\title{
Adaptive Neural Control for Nonaffine Pure-Feedback System Based on Extreme Learning Machine
}

\author{
Chenyang Xu $\mathbb{D}^{\text {D }}$, Humin Lei, Jiong Li, Jikun Ye $\mathbb{D}^{\circ}$, and Dongyang Zhang \\ Air and Missile Defence College, Air Force Engineering University, Xian 710051, China \\ Correspondence should be addressed to Chenyang Xu; 18729057322@163.com
}

Received 25 January 2019; Accepted 21 May 2019; Published 16 June 2019

Academic Editor: Sabri Arik

Copyright (C) 2019 Chenyang Xu et al. This is an open access article distributed under the Creative Commons Attribution License, which permits unrestricted use, distribution, and reproduction in any medium, provided the original work is properly cited.

\begin{abstract}
For nonaffine pure-feedback systems, an adaptive neural control method based on extreme learning machine (ELM) is proposed in this paper. Different from the existing methods, this scheme firstly converts the original system into a nonaffine system containing only one unknown term by equivalent transformation, thus avoiding the cumbersome and complex indirect design process of traditional backstepping methods. Secondly, a high-performance finite-time-convergence-differentiator (FD) is designed, through which the system state variables and their derivatives are accurately estimated to ensure the control effect. Thirdly, based on the implicit function theorem, the ELM neural network is introduced to approximate the uncertain items of the system, which simplifies the repeated adjustment process of the network training parameters. Meanwhile, the minimum learning parameter algorithm (MLP) is adopted to design the adaptive law for the norm of the network weight vector, which significantly reduces calculations. And it is theoretically proved that the closed-loop control system is stable and the tracking error is bounded. Finally, the effectiveness of the designed controller is verified by simulation.
\end{abstract}

\section{Introduction}

With the development of modern science and technology, automatic control theory has gradually formed a relatively mature theoretical system after evolving for more than half a century, and in particular, the linear control theory is becoming increasingly complete. However, various accurate analyses and experimental results indicate that all natural and practical engineering systems are nonlinear virtually [1]. Therefore, using the linear system theory to design controllers by ignoring the nonlinear factors of the actual system cannot meet the increasing control requirement of the nonlinear system. In recent years, the issue of the nonlinear control has attracted widespread attention from scholars in various countries and has achieved many breakthrough achievements [2-4].

In the research of the nonlinear control, the adaptive control has always been the focus. By combining the backstepping design [2] with the neural network's approximation ability [5], several adaptive neural control methods $[6,7]$ were proposed for the following strict-feedback nonlinear systems with affine forms:

$$
\begin{aligned}
\dot{x}_{i} & =f_{i}\left(\boldsymbol{x}_{i}\right)+g_{i}\left(\boldsymbol{x}_{i}\right) x_{i+1}, \quad i=1,2, \ldots n-1 \\
\dot{x}_{n} & =f_{n}\left(\boldsymbol{x}_{n}\right)+g_{n}\left(\boldsymbol{x}_{n}\right) u \\
y & =x_{1}
\end{aligned}
$$

where $\boldsymbol{x}_{i}=\left[x_{1}, \ldots, x_{i}\right]^{\mathrm{T}} \in \mathbf{R}^{i}(1 \leq i \leq n)$ is the state vector, $u$ and $y$ are the system input and the system output, respectively; $f_{i}(\bullet)$ and $g_{i}(\bullet)(1 \leq i \leq n)$ are unknown smooth functions. In [8], by introducing an improved Lyapunov function, a singularity-free adaptive neural backstepping controller was designed, which guaranteed the uniform ultimate boundness of the closed-loop adaptive system. For the uncertain multi-input/multioutput (MIMO) affine nonlinear system, an improved neural control scheme was proposed by using the block-triangular structure properties in [9], which avoided the controller's singularity problem. Based on the Radial Basis Function Neural Network (RBFNN), an adaptive backstepping controller was designed in [10], which theoretically guaranteed that the system output would converge to a small neighborhood of the desired trajectory. By analyzing the convergence of the tracking error with Barbalat's Lemma, a new type of adaptive backstepping neural controller was 
designed in [11], strengthening its robustness and making the accuracy of the ultimate tracking error determined. In order to avoid the complicated design of the control law in [810], an adaptive neural controller without backstepping was proposed in $[12,13]$, which greatly simplified the control law design process and enhanced the practicality of the control method.

Although the above methods in the literature have achieved certain control results, they are all aimed at strictfeedback systems. However, pure-feedback systems with nonaffine forms, such as chemical reaction, flight control, and biochemical process, are a type of more general and realistic description of practical nonlinear systems than strictfeedback systems [14]. Therefore, the research of the control problem for nonaffine pure-feedback system owns better engineering application value. For the pure-feedback system, a backstepping adaptive control method was proposed based on RBFNN in $[15,16]$, which ensured all signals of the closed-loop system were semiglobally uniformly ultimately bounded, but all aiming at inputs of affine forms. In [17], by designing an adaptive variable structure neural network control strategy, the stability of the system was proved. A cyclic indirect adaptive neural network controller for a purefeedback system was designed based on the mean value theorem in [18], which dealt with some of the challenging issues encountered in the complex nonlinear control systems. In [19], by transforming the nonaffine feedback system into an affine system with uncertainties based on the active disturbance rejection idea, an active disturbance rejection controller by employing the adaptive backstepping method was designed, which ensured the states could asymptotically converge to an arbitrary small region around zero. The hidden layer node parameters of RBFNN adopted in [15-19] were mainly determined by using prior knowledge, which will affect the tracking accuracy and the control effect of the control system [14]. In view of this, Huang et al. [20, 21] proposed the ELM fast learning algorithm, which was characterized by randomly determining the hidden layer training parameters. In the training process, only the output weights of the network needed to be adjusted; thus the learning speed was faster and the generalization performance was better. In [22], an adaptive backstepping control method based on ELM was proposed and applied to a dual-axis motion platform. A robust adaptive control method based on ELM was designed in [23], which was proved to be effective by applying it to a two-degree-of-freedom rigid robotic arm. On the basis of the backstepping method, an adaptive neural control method based on ELM was proposed in [14]. It was applied to the continuous stirred tank reactor (CSTR) system and achieved good control effect. However, [14-19, 22, 23] still did not get rid of the indirect design of the control law using the backstepping method, resulting in complicated control law design processes, complicated controller forms and complex parameter adjustments, which were not suitable for practical engineering applications.

The above researches on the control of pure-feedback systems mostly had three shortcomings. First, the cumbersome indirect design process of the backstepping design and the complex control law form restricted the engineering application of the researches. Second, the repetitive derivation of the deduced virtual control law was needed in the design process, which affected the control effect, especially when the system was subjected to strong external interference. Third, the adaptive law of the network weight vector were directly designed, which let the algorithm's real-time performance to be difficult to guarantee due to the large amount of calculations.

Based on the above shortages, this paper proposes a novel adaptive neural controller without the backstepping design by the equivalent conversion of a nonaffine pure-feedback system. By introducing ELM to accurately approximate unknown functions, the process of the repetitive adjustments of the hidden layer network parameters is avoided. The MLP algorithm is adopted to adaptively adjust the norm of the weight vector, which greatly reduces the amount of adaptive calculations. And through employing FD to achieve effective estimation of the system state variables, the control accuracy is ensured [24]. Simulation examples verify the effectiveness and the superiority of the design method. In general, the control method proposed in this paper has three advantages. First, compared with the traditional affine control method, the nonaffine control method designed in this paper better retains the key dynamic characteristics of the controlled object, and the control effect is more reliable and more practical. Secondly, the control method designed in this paper avoids the cumbersome process of the traditional backstepping control method, thus the virtual control law need not to be obtained step by step. The control law form is more concise. The control precision and the control robustness are also enhanced to some extent. Thirdly, the MLP algorithm is used to reduce the computational complexity of the neural network weights, ensuring the real-time performance of the proposed control method.

\section{Problem Formulation}

The pure-feedback system has the same triangular structure as the strict-feedback nonlinear system. The difference between them is that the structure of the strict-feedback nonlinear system is affine; that is, the mathematical expression of each state variable and control input is affine. However, the structure of the pure-feedback nonlinear system is nonaffine. Most engineering systems in practical applications are essentially nonaffine, so the study of nonaffine pure-feedback nonlinear systems has better engineering application value [14]. Consider the following nonaffine pure-feedback nonlinear system:

$$
\begin{aligned}
\dot{x}_{i} & =f_{i}\left(\bar{x}_{i}, x_{i+1}\right), \quad 1 \leq i \leq n-1 \\
\dot{x}_{n} & =f_{n}\left(\overline{\boldsymbol{x}}_{n}, u\right), \quad n \geq 2 \\
y & =x_{1}
\end{aligned}
$$

where $\overline{\boldsymbol{x}}_{i}=\left[x_{1}, x_{2}, \ldots, x_{i}\right]^{\mathrm{T}} \in \mathbf{R}^{n}$ and $\overline{\boldsymbol{x}}_{n}=\left[x_{1}, \ldots, x_{n}\right]^{\mathrm{T}} \in$ $\mathbf{R}^{n}$ are the system state vectors, $n$ is the order of the system, $i=1, \ldots, n-1, u \in \mathbf{R}$, and $y \in \mathbf{R}$ are the system input and the system output, respectively; $f_{i}(\bullet)$ and $f_{n}(\bullet)$ are unknown smooth nonlinear functions, which are 
continuously differentiable. It is apparent that the system is nonaffine for each state variable and control input.

The control goal of this paper is to design the control law $u$ to make all the signals of the closed-loop system semiglobally uniformly ultimately bounded, so that the system output $y$ can track the reference signal $y_{\mathrm{d}}$ stably. Before designing the controller, make the following assumption for system (2).

Assumption 1. For any $\left(\overline{\boldsymbol{x}}_{n}, u\right) \in \Omega_{\boldsymbol{x}} \times \mathbf{R}$, the following inequalities are established

$$
\begin{aligned}
& \frac{\partial f_{i}\left(\overline{\boldsymbol{x}}_{i}, x_{i+1}\right)}{\partial x_{i+1}} \neq 0, \quad i=1, \ldots, n-1 \\
& \frac{\partial f_{n}\left(\overline{\boldsymbol{x}}_{n}, u\right)}{\partial u} \neq 0
\end{aligned}
$$

where $\Omega_{\boldsymbol{x}} \in \mathbf{R}^{n}$ is a controllable area.

In order to avoid the cumbersome and the complicated design process of the traditional backstepping method in the control law design, the following equivalent transformation is performed on system (2).

Step 1. Let $z_{1}=x_{1}=y$.

Step 2. Let $z_{2}=\dot{z}_{1}=f_{1}\left(x_{1}, x_{2}\right)$. From (2), the time derivative of $z_{2}$ is derived as

$$
\begin{aligned}
\dot{z}_{2} & =\frac{\partial f_{1}\left(x_{1}, x_{2}\right)}{\partial x_{1}} \dot{x}_{1}+\frac{\partial f_{1}\left(x_{1}, x_{2}\right)}{\partial x_{2}} \dot{x}_{2} \\
& =\frac{\partial f_{1}\left(x_{1}, x_{2}\right)}{\partial x_{1}} f_{1}\left(x_{1}, x_{2}\right)+\frac{\partial f_{1}\left(x_{1}, x_{2}\right)}{\partial x_{2}} f_{2}\left(\bar{x}_{2}, x_{3}\right) \\
& \triangleq F_{1}\left(\bar{x}_{3}\right)
\end{aligned}
$$

and so on.

Step $i(3 \leq i \leq n-1)$. Let $z_{i}=\dot{z}_{i-1}=F_{i-2}\left(\overline{\boldsymbol{x}}_{i}\right)$. From (2), the time derivative of $z_{i}$ is derived as

$$
\begin{aligned}
\dot{z}_{i}= & \frac{\partial F_{i-2}\left(\overline{\boldsymbol{x}}_{i}\right)}{\partial x_{1}} \dot{x}_{1}+\frac{\partial F_{i-2}\left(\overline{\boldsymbol{x}}_{i}\right)}{\partial x_{1}} \dot{x}_{2}+\ldots+\frac{\partial F_{i-2}\left(\overline{\boldsymbol{x}}_{i}\right)}{\partial x_{i}} \dot{x}_{i} \\
= & \frac{\partial F_{i-2}\left(\overline{\boldsymbol{x}}_{i}\right)}{\partial x_{1}} f_{1}\left(\overline{\boldsymbol{x}}_{2}\right)+\frac{\partial F_{i-2}\left(\overline{\boldsymbol{x}}_{i}\right)}{\partial x_{2}} f_{2}\left(\overline{\boldsymbol{x}}_{3}\right)+\ldots \\
& +\frac{\partial F_{i-2}\left(\overline{\boldsymbol{x}}_{i}\right)}{\partial x_{i}} f_{i}\left(\overline{\boldsymbol{x}}_{i+1}\right) \triangleq F_{i-1}\left(\overline{\boldsymbol{x}}_{i+1}\right)
\end{aligned}
$$

Step $n$. Let $z_{n}=\dot{z}_{n-1}=F_{n-2}\left(\overline{\boldsymbol{x}}_{n}\right)$. From (2), the time derivative of $z_{n}$ is derived as

$$
\begin{aligned}
\dot{z}_{n}= & \frac{\partial F_{n-2}\left(\overline{\boldsymbol{x}}_{n}\right)}{\partial x_{1}} \dot{x}_{1}+\frac{\partial F_{n-2}\left(\overline{\boldsymbol{x}}_{n}\right)}{\partial x_{1}} \dot{x}_{2}+\ldots \\
& +\frac{\partial F_{n-2}\left(\overline{\boldsymbol{x}}_{n}\right)}{\partial x_{n}} \dot{x}_{n}
\end{aligned}
$$

$$
\begin{aligned}
= & \frac{\partial F_{n-2}\left(\overline{\boldsymbol{x}}_{n}\right)}{\partial x_{1}} f_{1}\left(\overline{\boldsymbol{x}}_{2}\right)+\frac{\partial F_{n-2}\left(\overline{\boldsymbol{x}}_{n}\right)}{\partial x_{2}} f_{2}\left(\overline{\boldsymbol{x}}_{3}\right)+\ldots \\
& +\frac{\partial F_{n-2}\left(\overline{\boldsymbol{x}}_{n}\right)}{\partial x_{n}} f_{n}\left(\overline{\boldsymbol{x}}_{n}, u\right) \triangleq F_{n-1}\left(\overline{\boldsymbol{x}}_{n}, u\right)
\end{aligned}
$$

After the above transformation, (2) is as follows:

$$
\begin{gathered}
\dot{z}_{1}=z_{2} \\
\dot{z}_{2}=z_{3} \\
\vdots \\
\dot{z}_{n}=F_{n-1}\left(\overline{\boldsymbol{x}}_{n}, u\right) \\
y=z_{1}
\end{gathered}
$$

Combining (2) with Assumption 1, it is evident that $F_{n-1}\left(\overline{\boldsymbol{x}}_{n}, u\right)$ is a completely unknown continuously differentiable function and has the following theorem.

Theorem 2. Under the premise of Assumption 1, after the transformation of Steps 1 n, there is always

$$
\frac{\partial F_{n-1}\left(\overline{\boldsymbol{x}}_{n}, u\right)}{\partial u} \neq 0
$$

Proof. From Steps $1 \sim n$, it can be known that

$$
\begin{aligned}
& \frac{\partial F_{n-1}\left(\overline{\boldsymbol{x}}_{n}, u\right)}{\partial u}=\frac{\partial F_{n-2}\left(\overline{\boldsymbol{x}}_{n}\right)}{\partial x_{n}} \cdot \frac{\partial f_{n}\left(\overline{\boldsymbol{x}}_{n}, u\right)}{\partial u} \\
& =\frac{\partial F_{n-3}\left(\overline{\boldsymbol{x}}_{n-1}\right)}{\partial x_{n-1}} \cdot \frac{\partial f_{n-1}\left(\overline{\boldsymbol{x}}_{n}\right)}{\partial x_{n}} \cdot \frac{\partial f_{n}\left(\overline{\boldsymbol{x}}_{n}, u\right)}{\partial u} \\
& =\frac{\partial F_{n-4}\left(\overline{\boldsymbol{x}}_{n-2}\right)}{\partial x_{n-2}} \cdot \frac{\partial f_{n-2}\left(\overline{\boldsymbol{x}}_{n-1}\right)}{\partial x_{n-1}} \cdot \frac{\partial f_{n-1}\left(\overline{\boldsymbol{x}}_{n}\right)}{\partial x_{n}} \\
& \quad \frac{\partial f_{n}\left(\overline{\boldsymbol{x}}_{n}, u\right)}{\partial u} \\
& =\prod_{i=1}^{n-1} \frac{\partial f_{i}\left(\overline{\boldsymbol{x}}_{i+1}\right)}{\partial x_{i+1}} \cdot \frac{\partial f_{n}\left(\overline{\boldsymbol{x}}_{n}, u\right)}{\partial u}
\end{aligned}
$$

Then according to Assumption 1, it is easy to obtain $\partial F_{n-1}\left(\overline{\boldsymbol{x}}_{n}, u\right) / \partial u \neq 0$.

Remark 3. Compared with system (2), system (7) not only has a simpler form, but also contains only one unknown function. When the control law is designed based on this system, the complicated design process of the traditional backstepping method is no longer needed.

At this point, the control target can be translated into the following: for the pure-feedback nonaffine system shown in (7), design the control law $u$ to make $z_{1}$ stably track the reference signal $y_{\mathrm{d}}$. 


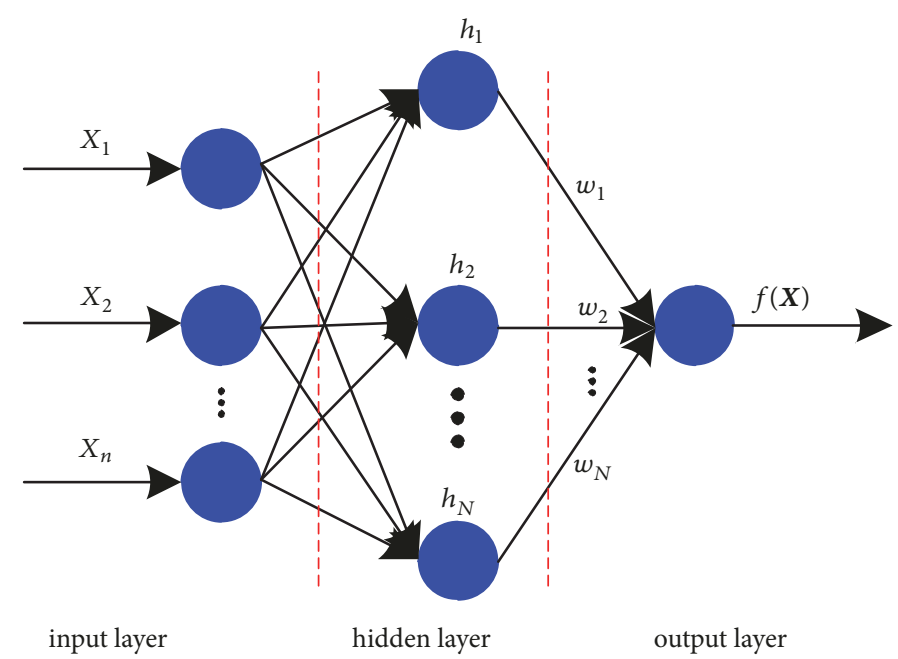

FIGURE 1: The basic structure of a SLFN.

\section{Extreme Learning Machine (ELM)}

3.1. The Basic Concept of ELM. In this paper, an extreme learning machine (ELM) is employed to approximate an unknown continuous function. Extreme learning machine is a simple and practical single hidden layer feedforward neural network (SLFN) learning algorithm. ELM differs from the traditional neural network learning algorithm (such as RBF algorithm). It only needs to set the number of the hidden layer nodes in the network. The algorithm can generate a unique optimal solution without adjusting the training parameters during the execution of the algorithm, which has a fast learning speed and a good ability in generalization.

For a single-output SLFN with $N$ hidden layer nodes, the basic structure is shown in Figure 1.

The mathematical description of the network output is

$$
f(\boldsymbol{X})=\boldsymbol{W}^{\mathrm{T}} \boldsymbol{h}\left(\boldsymbol{X}, \boldsymbol{\omega}_{i}, b_{i}\right)
$$

where $\boldsymbol{X}=\left[X_{1}, X_{2}, \ldots, X_{n}\right]^{\mathrm{T}} \in \mathbf{R}^{n}$ is the input vector, $\boldsymbol{W}=$ $\left[w_{1}, w_{2}, \ldots, w_{N}\right]^{\mathrm{T}} \in \mathbf{R}^{N}$ is the weight vector $(N$ is the number of the hidden layer nodes), $\boldsymbol{h}=\left[h_{1}, h_{2}, \ldots, h_{N}\right]^{\mathrm{T}} \in \mathbf{R}^{N}$ is the activation function, $\boldsymbol{\omega}_{i}=\left[\omega_{i 1}, \omega_{i 2}, \ldots, \omega_{i n}\right]^{\mathrm{T}} \in \mathbf{R}^{n}$ and $b_{i}(i=$ $1,2, \ldots, N)$ stand for the training parameters of the network, and $\boldsymbol{h}\left(\boldsymbol{X}, \boldsymbol{\omega}_{i}, b_{i}\right)$ is determined by the type of the hidden layer node.

When the hidden layer node is an additive Sigmoid activation function

$$
h_{i}\left(\boldsymbol{X}, \boldsymbol{\omega}_{i}, b_{i}\right)=\frac{1}{1+\exp \left(-\left(\boldsymbol{X}^{\mathrm{T}} \cdot \boldsymbol{\omega}_{i}+b_{i}\right)\right)}
$$

When it is an RBF activation function

$$
h_{i}\left(\boldsymbol{X}, \boldsymbol{\omega}_{i}, b_{i}\right)=\exp \left(-b_{i}\left\|\boldsymbol{X}-\boldsymbol{\varpi}_{i}\right\|^{2}\right)
$$

When it is a Hard-limit activation function

$$
h_{i}\left(\boldsymbol{X}, \boldsymbol{\omega}_{i}, b_{i}\right)= \begin{cases}1, & \boldsymbol{X}^{\mathrm{T}} \cdot \boldsymbol{\omega}_{i}-b_{i} \geq 0 \\ 0, & \boldsymbol{X}^{\mathrm{T}} \cdot \boldsymbol{\omega}_{i}-b_{i}<0\end{cases}
$$

3.2. The Learning Principle of ELM. For the above singleoutput SLFN, if any $M$ sets of data are given, $\left(\boldsymbol{v}_{j}, t_{j}\right) \in \mathbf{R}^{n} \times \mathbf{R}$, where $\boldsymbol{v}_{j}=\left[v_{j 1}, v_{j 2}, \ldots, v_{j n}\right]^{\mathrm{T}}, j=1,2, \ldots, M$, and $n$ is the input vector dimension, then the function model of the SLFN containing $N$ hidden layer nodes is as follows:

$$
\begin{aligned}
f\left(\boldsymbol{v}_{j}\right)=\boldsymbol{W}^{\mathrm{T}} \boldsymbol{h}\left(\boldsymbol{v}_{j}, \boldsymbol{\omega}_{i}, b_{i}\right)= & \tau_{j}, \\
& j=1, \ldots, M ; i=1, \ldots, N
\end{aligned}
$$

Write the above formula as a matrix

$$
H W=\tau
$$

where

$$
\begin{aligned}
\boldsymbol{H} & =\left[\begin{array}{c}
\boldsymbol{h}\left(\boldsymbol{v}_{1}, \boldsymbol{\omega}_{i}, b_{i}\right) \\
\vdots \\
\boldsymbol{h}\left(\boldsymbol{v}_{M}, \boldsymbol{\omega}_{i}, b_{i}\right)
\end{array}\right] \\
& =\left[\begin{array}{ccc}
h_{1}\left(\boldsymbol{v}_{1}, \boldsymbol{\omega}_{1}, b_{1}\right) & \cdots & h_{N}\left(\boldsymbol{v}_{1}, \boldsymbol{\omega}_{N}, b_{N}\right) \\
\vdots & \ddots & \vdots \\
h_{1}\left(\boldsymbol{v}_{M}, \boldsymbol{\omega}_{1}, b_{1}\right) & \cdots & h_{N}\left(\boldsymbol{v}_{M}, \boldsymbol{\omega}_{N}, b_{N}\right)
\end{array}\right]_{M \times N} \\
\boldsymbol{W} & =\left[\begin{array}{c}
w_{1} \\
\vdots \\
w_{N}
\end{array}\right]_{N \times 1},
\end{aligned}
$$

$$
\boldsymbol{\tau}=\left[\begin{array}{c}
\tau_{1} \\
\vdots \\
\tau_{M}
\end{array}\right]_{M \times 1}
$$

If $\boldsymbol{h}\left(\boldsymbol{v}_{j}, \boldsymbol{\omega}_{i}, b_{i}\right)$ is infinitely derivable on any interval, according to [15], when the number of samples $M$ is bigger 
than the number of nodes $N$ in the hidden layer, the SLFN can approximate the training sample with minimal errors. By training the neural network, $\widehat{\boldsymbol{W}}, \widehat{\boldsymbol{\omega}}_{i}$, and $\widehat{b}_{i}$ can be obtained that satisfy

$$
\left\|\boldsymbol{H}\left(\widehat{\boldsymbol{\omega}}_{i}, \widehat{b}_{i}\right) \widehat{\boldsymbol{W}}-\boldsymbol{t}\right\|=\min _{\boldsymbol{\varpi}_{i}, b_{i}, \boldsymbol{W}}\left\|\boldsymbol{H}\left(\boldsymbol{\omega}_{i}, b_{i}\right) \boldsymbol{W}-\boldsymbol{t}\right\|
$$

where $\boldsymbol{t}=\left[t_{1}, t_{2}, \ldots, t_{M}\right]^{\mathrm{T}}$ is the given training sample value.

Remark 4. The traditional algorithms based on the gradient descent need to preliminarily set the training parameters $\widehat{\boldsymbol{\omega}}_{i}$ and $\widehat{b}_{i}$ of the network according to experience and continuously adjust them during the iteration process. However, using the ELM algorithm, $\widehat{\widehat{\boldsymbol{\omega}}}_{i}$ and $\widehat{b}_{i}$ are randomly determined, and the task is only to solve the least-squares solution of the weight vector $\widehat{W}$ of (17).

3.3. The Approximation to Unknown Functions of ELM. For any unknown nonlinear continuous function $F(\boldsymbol{X})$, using the ELM algorithm, selecting enough nodes (selecting a sufficiently large $N$ ), and following the universal approximation theorem [5], there must be an ideal weight vector $\boldsymbol{W}^{*}=$ $\left[w_{1}{ }^{*}, w_{2}{ }^{*}, \ldots, w_{N}{ }^{*}\right]^{\mathrm{T}} \in \mathbf{R}^{N}$ that satisfy

$$
F(\boldsymbol{X})=\boldsymbol{W}^{* \mathrm{~T}} \boldsymbol{h}(\boldsymbol{X})+\varepsilon, \quad|\varepsilon| \leq \varepsilon_{M}
$$

where $\varepsilon \in \mathbf{R}$ is approximation error, $\varepsilon_{M} \in \mathbf{R}^{+}$is the upper bound of the approximation error. When taking $N$ large enough, $\varepsilon_{M}$ can be arbitrarily small [5].

Under the ELM algorithm, an effective approximation to $F(\boldsymbol{X})$ can be achieved simply by adjusting the weight vector $\boldsymbol{W}=\left[w_{1}, w_{2}, \ldots, w_{N}\right]^{\mathrm{T}}$ online. And based on the Lyapunov stability theory, an adaptive law that can make the closed-loop system stable is designed for $\boldsymbol{W}$, so that the boundedness and convergence of the approximation error can be guaranteed.

\section{Controller Design}

4.1. Adaptive Neural Control Law Design. For system (7), define the system output tracking error $e$ and the error function $E$

$$
\begin{aligned}
& e=y-y_{\mathrm{d}}=z_{1}-y_{\mathrm{d}} \\
& E=\left(\frac{\mathrm{d}}{\mathrm{d} t}+\lambda\right)^{n-1} e=\left[\begin{array}{ll}
\boldsymbol{\Lambda}^{\mathrm{T}} & 1
\end{array}\right] \boldsymbol{e}
\end{aligned}
$$

where $y_{\mathrm{d}}$ is the reference signal, $\lambda \in \mathbf{R}^{+}$is the design parameter, $n$ is the order of the system, $\boldsymbol{\Lambda}=\left[\lambda^{n-1},(n-\right.$ 1) $\left.\lambda^{n-2}, \ldots,(n-1) \lambda\right]^{\mathrm{T}}$, and $\boldsymbol{e}=\left[e, \dot{e}, \ldots, e^{(n-1)}\right]^{\mathrm{T}}$. Since $(s+\lambda)^{3}$ is a Hurwitz polynomial, when $E$ is bounded, $e$ must be bounded.

Combining (19) and (7), $\boldsymbol{e}$ can also be expressed as

$$
e=z-y_{d}
$$

where

$$
\begin{aligned}
\boldsymbol{z} & =\left[z_{1}, z_{2}, \ldots, z_{n}\right]^{\mathrm{T}} \\
\boldsymbol{y}_{\boldsymbol{d}} & =\left[y_{d}, \dot{y}_{d}, \ldots, y_{d}{ }^{(n-1)}\right]^{\mathrm{T}}
\end{aligned}
$$

From (19), the time derivative of $E$ is derived as

$$
\dot{E}=\left[\begin{array}{ll}
0 & \Lambda^{\mathrm{T}}
\end{array}\right] \boldsymbol{e}+\left(\dot{z}_{n}-y_{\mathrm{d}}^{(n)}\right)
$$

Substitute (7) into (22)

$$
\dot{E}=F_{n-1}\left(\overline{\boldsymbol{x}}_{n}, u\right)-y_{\mathrm{d}}{ }^{(n)}+\left[\begin{array}{ll}
0 & \boldsymbol{\Lambda}^{\mathrm{T}}
\end{array}\right] \boldsymbol{e}
$$

Consider that the influence of the external disturbances in the actual engineering system cannot be ignored, so if the state variable information $\left(\boldsymbol{z}=\left[z_{1}, z_{2}, \ldots, z_{n}\right]^{\mathrm{T}}\right)$ of the system (7) is directly extracted, a large amount of random noise is bound to be introduced, which is unfavorable to the design of the control law and it is difficult to ensure the stability of the closed-loop control system. A new finite-time-convergencedifferentiator (FD) will be used below to effectively filter out the noise information in $z$ for accurate estimation. The estimated value of $z$ is expressed as follows:

$$
\widehat{z}=\left[\widehat{z}_{1}, \widehat{z}_{2}, \ldots, \widehat{z}_{n}\right]^{\mathrm{T}}
$$

Substitute (24) into (20) and obtain the estimation of $\boldsymbol{e}$

$$
\widehat{\boldsymbol{e}}=\widehat{z}-\boldsymbol{y}_{\mathrm{d}}
$$

Replace $\boldsymbol{e}$ in (23) with $\widehat{\boldsymbol{e}}$

$$
\dot{\hat{E}}=F_{n-1}\left(\overline{\boldsymbol{x}}_{n}, u\right)-y_{\mathrm{d}}{ }^{(n)}+\left[\begin{array}{ll}
0 & \Lambda^{\mathrm{T}}
\end{array}\right] \widehat{\boldsymbol{e}}
$$

Let

$$
\Phi\left(\overline{\boldsymbol{x}}_{n}, u\right)=F_{n-1}\left(\overline{\boldsymbol{x}}_{n}, u\right)-k_{1} u
$$

where $k_{1} \in \mathbf{R}^{+}$is design parameter.

Combine (26) and (27)

$$
\begin{aligned}
\dot{\widehat{E}} & =\Phi\left(\overline{\boldsymbol{x}}_{n}, u\right)+k_{1} u-y_{\mathrm{d}}{ }^{(n)}+\left[\begin{array}{ll}
0 & \Lambda^{\mathrm{T}}
\end{array}\right] \widehat{\boldsymbol{e}} \\
& =\Phi\left(\overline{\boldsymbol{x}}_{n}, u\right)+k_{1} u+v
\end{aligned}
$$

where

$$
v=-y_{\mathrm{d}}^{(n)}+\left[\begin{array}{ll}
0 & \boldsymbol{\Lambda}^{\mathrm{T}}
\end{array}\right] \widehat{\boldsymbol{e}}
$$

Design the control law $u$ as

$$
u=k_{1}^{-1}\left(u_{0}-u_{1}\right)
$$

where

$$
u_{0}=-k_{2} \widehat{E}-k_{3} \int_{0}^{t} \widehat{E} \mathrm{~d} \tau-v
$$

In this paper, ELM is used to design $u_{1}$ to offset the influence of $\Phi\left(\overline{\boldsymbol{x}}_{n}, u\right)$. In order to facilitate the subsequent design process, the implicit function theorem is introduced here [25].

Theorem 5. Assume that the implicit function $\Psi: \mathbf{R}^{l} \times \mathbf{R}^{r} \longrightarrow$ $\mathbf{R}^{l}$ is continuously differentiable at each point $(\varsigma, \sigma)$ on the open set $Y \subset \mathbf{R}^{l} \times \mathbf{R}^{r} .\left(\varsigma_{0}, \sigma_{0}\right)$ is a point in $Y . \Psi\left(\varsigma_{0}, \sigma_{0}\right)=0$ and the Jacobian matrix $(\partial \Psi / \partial \varsigma)\left(\varsigma_{0}, \sigma_{0}\right)$ is nonsingular. Then, for any $\sigma \in G$, a neighborhood $U \subset \mathbf{R}^{l}$ of $\varsigma_{0}$ and a neighborhood $G \subset$ $\mathbf{R}^{r}$ of $\sigma_{0}$ can make the equation $\Psi(\varsigma, \sigma)=0$ which has a unique solution $\varsigma \in U$. The solution can be expressed as $\varsigma=g_{0}(\sigma)$, where $g_{0}(\bullet)$ is a continuously differentiable function on $\sigma=\sigma_{0}$. 
Remark 6. Theorem 5 shows that once the implicit function $\Psi(\varsigma, \sigma)$ satisfies all conditions in the theorem, $\varsigma$ can be expressed as a continuously differentiable function of $\sigma$, that is, $\varsigma=g_{0}(\sigma)$. At this point, using ELM to approximate $\Psi(\varsigma, \sigma)$, only $\sigma$ is needed as the input signal of the neural network to obtain a satisfactory approximation effect. This is where the special meaning of the implicit function theorem lies [24].

Let

$$
\begin{aligned}
\Gamma\left(\overline{\boldsymbol{x}}_{n}, u_{0}, u_{1}\right) & \triangleq \Phi\left(\overline{\boldsymbol{x}}_{n}, u\right)-u_{1} \\
& =\Phi\left(\overline{\boldsymbol{x}}_{n}, k_{1}^{-1}\left(u_{0}-u_{1}\right)\right)-u_{1}
\end{aligned}
$$

To illustrate that $\Gamma\left(\overline{\boldsymbol{x}}_{n}, u_{0}, u_{1}\right)$ satisfies the implicit function theorem, the following theorem is given.

\section{Theorem 7. Define}

$$
k_{1}>\frac{1}{2} \frac{\partial F_{n-1}\left(\overline{\boldsymbol{x}}_{n}, u\right)}{\partial u}
$$

Then there are a controllable area $\Omega_{\mathrm{x}} \subset \mathbf{R}^{n}$ and a unique $u_{1}{ }^{*}$ for any $\left(\overline{\boldsymbol{x}}_{n}, u\right) \in \Omega_{\mathrm{x}} \times \mathbf{R}, u_{1}{ }^{*}$ satisfies

$$
\Gamma\left(\overline{\boldsymbol{x}}_{n}, u_{0}, u_{1}^{*}\right)=0
$$

Proof. According to $[25,26]$, a sufficient condition for the existence of $u_{1}{ }^{*}$ is that the following inequality holds

$$
\left|\frac{\partial \Phi\left(\bar{x}_{n}, u\right)}{\partial u_{1}{ }^{*}}\right|<1
$$

Considering (8), (30), and (33), there are

$$
\begin{aligned}
\left|\frac{\partial \Phi\left(\overline{\boldsymbol{x}}_{n}, u\right)}{\partial u_{1}{ }^{*}}\right| & =\left|\frac{\partial\left[F_{n-1}\left(\overline{\boldsymbol{x}}_{n}, u\right)-k_{1} u\right]}{\partial u_{1}{ }^{*}}\right| \\
& =\left|\frac{\partial\left[F_{n-1}\left(\overline{\boldsymbol{x}}_{n}, u\right)-k_{1} u\right]}{\partial u} \frac{\partial u}{\partial u_{1}{ }^{*}}\right| \\
& =\left|\left[\frac{\partial F_{n-1}\left(\overline{\boldsymbol{x}}_{n}, u\right)}{\partial u}-k_{1}\right] \frac{1}{k_{1}}\right| \\
& =\left|\frac{1}{k_{1}} \frac{\partial F_{n-1}\left(\overline{\boldsymbol{x}}_{n}, u\right)}{\partial u}-1\right|<1
\end{aligned}
$$

Therefore, $u_{1}{ }^{*}$ exists.

Further

$$
\begin{aligned}
\frac{\partial \Gamma\left(\overline{\boldsymbol{x}}_{n}, u_{0}, u_{1}{ }^{*}\right)}{\partial u_{1}{ }^{*}} & =\frac{\partial\left[\Phi\left(\overline{\boldsymbol{x}}_{n}, u^{*}\right)-u_{1}{ }^{*}\right]}{\partial u_{1}{ }^{*}} \\
& =\frac{\partial\left[F_{n-1}\left(\overline{\boldsymbol{x}}_{n}, u^{*}\right)-k_{1} u^{*}\right]}{\partial u_{1}{ }^{*}}-1 \\
& =\frac{\partial\left[F_{n-1}\left(\overline{\boldsymbol{x}}_{n}, u^{*}\right)-k_{1} u^{*}\right]}{\partial u^{*}} \frac{\partial u^{*}}{\partial u_{1}{ }^{*}}-1 \\
& =\left[\frac{\partial F_{n-1}\left(\overline{\boldsymbol{x}}_{n}, u^{*}\right)}{\partial u^{*}}-k_{1}\right]\left(-\frac{1}{k_{1}}\right)-1 \\
& =-\frac{1}{k_{1}} \frac{\partial F_{n-1}\left(\overline{\boldsymbol{x}}_{n}, u^{*}\right)}{\partial u^{*}}
\end{aligned}
$$

where $u^{*}=k_{1}^{-1}\left(u_{0}-u_{1}{ }^{*}\right)$.
Combine (8) and (37)

$$
\frac{\partial \Gamma\left(\overline{\boldsymbol{x}}_{n}, u_{0}, u_{1}{ }^{*}\right)}{\partial u_{1}{ }^{*}} \neq 0
$$

According to Theorem 7 and (38), $\Gamma\left(\overline{\boldsymbol{x}}_{n}, u_{0}, u_{1}\right)$ satisfies the implicit function theorem. Therefore, $u_{1}$ can be regarded as a function of $u_{0}$ and $\overline{\boldsymbol{x}}_{n}$, and further $\Phi\left(\overline{\boldsymbol{x}}_{n}, u\right)$ can be regarded as a function of $u_{0}$ and $\overline{\boldsymbol{x}}_{n}$.

Define $\boldsymbol{X}_{\text {in }}=\left[\overline{\boldsymbol{x}}_{n}{ }^{\mathrm{T}}, u_{0}\right]^{\mathrm{T}} \in \mathbf{R}^{n+1}$ as the input vector of ELM and introduce ELM to approximate $\Phi\left(\overline{\boldsymbol{x}}_{n}, u\right)$

$$
\Phi\left(\overline{\boldsymbol{x}}_{n}, u\right)=\boldsymbol{W}_{\Phi}{ }^{* \mathrm{~T}} \boldsymbol{h}\left(\boldsymbol{X}_{\mathrm{in}}\right)+\varepsilon, \quad|\varepsilon| \leq \varepsilon_{M}
$$

where $\boldsymbol{W}_{\Phi}{ }^{* \mathrm{~T}}=\left[w_{\Phi 1}^{*}, w_{\Phi 2}^{*}, \ldots, w_{\Phi p}^{*}\right]^{\mathrm{T}} \in \mathbf{R}^{p}$ is the weight vector; $p$ is the number of nodes; $\varepsilon$ and $\varepsilon_{M}$ are approximation errors and its upper bounds, respectively; and $\boldsymbol{h}\left(\boldsymbol{X}_{\text {in }}\right)=$ $\left[h_{1}\left(\boldsymbol{X}_{i n}\right), h_{2}\left(\boldsymbol{X}_{i n}\right), \ldots h_{p}\left(\boldsymbol{X}_{\text {in }}\right)\right]^{\mathrm{T}} \in \mathbf{R}^{p}$, where $h_{i}\left(\boldsymbol{X}_{\mathrm{in}}\right)(i=$ $1,2, \ldots, p)$ is the activation function. The following is based on the MLP idea to adaptively adjust the norm of the ELM weight vector.

Define $\omega=\left\|\boldsymbol{W}_{\Phi}{ }^{*}\right\|^{2}$ and design $u_{1}$ as

$$
u_{1}=\frac{1}{2} \widehat{E} \widehat{\omega} \boldsymbol{h}^{\mathrm{T}}\left(\boldsymbol{X}_{\text {in }}\right) \boldsymbol{h}\left(\boldsymbol{X}_{\text {in }}\right)
$$

where $\widehat{\omega}$ is the estimation of $\omega$ and its adaptive law is designed as

$$
\dot{\hat{\omega}}=\frac{\mu}{2} \widehat{E}^{2} \boldsymbol{h}^{\mathrm{T}}\left(\boldsymbol{X}_{\mathrm{in}}\right) \boldsymbol{h}\left(\boldsymbol{X}_{\mathrm{in}}\right)-2 \widehat{\omega}
$$

where $\mu \in \mathbf{R}^{+}$is the design parameter.

Substitute (31) and (40) into (30) and finally get the control law $u$

$$
\begin{aligned}
u= & k_{1}^{-1}\left(-k_{2} \widehat{E}-k_{3} \int_{0}^{t} \widehat{E} \mathrm{~d} \tau-v\right. \\
& \left.-\frac{1}{2} \widehat{E} \widehat{\omega} \boldsymbol{h}^{\mathrm{T}}\left(\boldsymbol{X}_{\text {in }}\right) \boldsymbol{h}\left(\boldsymbol{X}_{\text {in }}\right)\right)
\end{aligned}
$$

Remark 8. Different from $[15,16]$ in which the weight vector of the neural network is directly adjusted online, this paper regards $\boldsymbol{W}_{\Phi}{ }^{*}$ as a whole based on the MLP idea. Adaptive adjustment of $\omega$ requires only one online learning parameter $\widehat{\omega}$, and the computational complexity of the approximation algorithm is significantly reduced.

\subsection{Finite-Time-Convergence-Differentiator (FD) Design}

Theorem 9. Consider the following FD:

$$
\begin{aligned}
& \dot{\xi}_{1}=\xi_{2} \\
& \dot{\xi}_{2}=\xi_{3} \\
& \dot{\xi}_{n-1}=\xi_{n}
\end{aligned}
$$




$$
\begin{aligned}
\dot{\xi}_{n} & =R^{n}\left[-a_{1} \arctan \left(\xi_{1}-v(t)\right)-a_{2} \arctan \left(\frac{\xi_{2}}{R}\right)\right. \\
& \left.-\cdots-a_{n} \arctan \left(\frac{\xi_{n}}{R^{n-1}}\right)\right]
\end{aligned}
$$

where $R, a_{i}(i=1,2, \cdots, n) \in \mathbf{R}^{+}$stand for design parameters. There are $\phi>0$ and $\iota \phi>n$ so that

$$
\xi_{i}-v^{(i-1)}(t)=O\left(\left(\frac{1}{R}\right)^{\iota \phi-i+1}\right), \quad i=1,2, \ldots, n
$$

where $O\left((1 / R)^{i \phi-i+1}\right)$ denotes the order of the approximation between $\xi_{i}$ and $v^{(i-1)}(t)$ is $(1 / R)^{\tau \phi-i+1}, \phi=(1-\vartheta) / \vartheta, \vartheta \in$ $(0, \min \{\iota /(\iota+n), 1 / 2\}), n \geq 2$.

Proof. The detailed proof process of Theorem 9 can be found in [27].

Remark 10. In (43), $\xi_{i}(i=1,2, \ldots, n)$ is the state variable of the system, $\xi_{1}$ is the estimation of $v(t)$, and $\xi_{i}(i=2,3, \ldots, n)$ is the estimation of the $i-1$ th derivative of $v(t)$. At the same time, (44) shows that the estimation error is a high order infinitesimal of $(1 / R)^{\tau \phi-i+1}$.

FD can not only achieve accurate approximation of high-order differential signals, but also ensure smoother estimation when the system is disturbed by noise, which has certain advantages over traditional differentiators [27].

Use the above FD to estimate the state variable $z$ of system (7)

$$
\begin{aligned}
& \dot{\vec{z}}_{1}=\widehat{z}_{2} \\
& \dot{\vec{z}}_{2}=\widehat{z}_{3} \\
& \vdots \\
& \dot{\vec{z}}_{n-1}=\widehat{z}_{n} \\
& \dot{\vec{z}}_{n}=R^{n}\left[-a_{1} \arctan \left(\widehat{z}_{1}-y\right)-a_{2} \arctan \left(\frac{\widehat{z}_{2}}{R}\right)-\cdots\right. \\
& \left.\quad-a_{n} \arctan \left(\frac{\widehat{z}_{n}}{R^{n-1}}\right)\right]
\end{aligned}
$$

where $R \in \mathbf{R}^{+}$and $a_{i}(i=1,2, \cdots, n) \in \mathbf{R}^{+}$are parameters and $y$ stands for the output of system (7).

\subsection{Stability Analysis}

Theorem 11. For a nonaffine pure-feedback system (7), under the premise of Assumption 1, when the control law (42), the adaptive law (41), and the FD (45) are used, the closed-loop control system is semiglobally uniformly ultimately bounded.
Proof. Define the estimation error

$$
\begin{aligned}
& \widetilde{\boldsymbol{e}}=\widehat{\boldsymbol{e}}-\boldsymbol{e}=\widehat{z}-\boldsymbol{z} \\
& \widetilde{\omega}=\widehat{\omega}-\omega
\end{aligned}
$$

According to Theorem 9, there is $\delta_{M} \in \mathbf{R}^{+}$

$$
\|\widetilde{\boldsymbol{e}}\|=\|\widehat{z}-z\| \leq \delta_{M}
$$

Substitute (39) and (42) into (28)

$$
\begin{aligned}
\dot{\widehat{E}}= & -k_{2} \widehat{E}-k_{3} \int_{0}^{t} \widehat{E} \mathrm{~d} \tau-\frac{1}{2} \widehat{E} \widehat{\omega} \boldsymbol{h}^{\mathrm{T}}\left(\boldsymbol{X}_{\text {in }}\right) \boldsymbol{h}\left(\boldsymbol{X}_{\text {in }}\right) \\
& +\boldsymbol{W}_{\Phi}{ }^{* \mathrm{~T}} \boldsymbol{h}\left(\boldsymbol{X}_{\text {in }}\right)+\varepsilon
\end{aligned}
$$

Choose the following Lyapunov function candidate

$$
L=\frac{1}{2} \widehat{E}^{2}+\frac{1}{2} k_{3}\left(\int_{0}^{t} \widehat{E} \mathrm{~d} \tau\right)^{2}+\frac{\widetilde{\omega}^{2}}{2 \mu}
$$

Taking time derivative of (49) and invoking (41), (46), and (48) yield

$$
\begin{aligned}
\dot{L} & =\widehat{E} \dot{\widehat{E}}+k_{3} \widehat{E} \int_{0}^{t} \widehat{E} \mathrm{~d} \tau+\frac{\widetilde{\omega} \dot{\hat{\omega}}}{\mu}=\widehat{E}\left[-k_{2} \widehat{E}-k_{3} \int_{0}^{t} \widehat{E} \mathrm{~d} \tau\right. \\
& \left.-\frac{1}{2} \widehat{E} \widehat{\omega} \boldsymbol{h}^{\mathrm{T}}\left(\boldsymbol{X}_{\mathrm{in}}\right) \boldsymbol{h}\left(\boldsymbol{X}_{\mathrm{in}}\right)+\boldsymbol{W}_{\Phi}^{* \mathrm{~T}} \boldsymbol{h}\left(\boldsymbol{X}_{\mathrm{in}}\right)+\varepsilon\right] \\
& +k_{3} \widehat{E} \int_{0}^{t} \widehat{E} \mathrm{~d} \tau+\frac{\widetilde{\omega}}{\mu}\left[\frac{\mu}{2} \widehat{E}^{2} \boldsymbol{h}^{\mathrm{T}}\left(\boldsymbol{X}_{\mathrm{in}}\right) \boldsymbol{h}\left(\boldsymbol{X}_{\mathrm{in}}\right)-2 \widehat{\omega}\right] \\
& =-k_{2} \widehat{E}^{2}-\frac{1}{2} \widehat{E}^{2} \omega \boldsymbol{h}^{\mathrm{T}}\left(\boldsymbol{X}_{\mathrm{in}}\right) \boldsymbol{h}\left(\boldsymbol{X}_{\mathrm{in}}\right) \\
& +\widehat{E} \boldsymbol{W}_{\Phi}{ }^{* \mathrm{~T}} \boldsymbol{h}\left(\boldsymbol{X}_{\mathrm{in}}\right)+\widehat{E} \varepsilon-\frac{2 \widetilde{\omega} \widehat{\omega}}{\mu}
\end{aligned}
$$

Notice that

$$
\begin{aligned}
\widehat{E} \boldsymbol{W}_{\Phi}{ }^{* \mathrm{~T}} \boldsymbol{h}\left(\boldsymbol{X}_{\text {in }}\right) & \leq \frac{\widehat{E}^{2}}{2}\left\|\boldsymbol{W}_{\Phi}{ }^{* \mathrm{~T}} \boldsymbol{h}\left(\boldsymbol{X}_{\text {in }}\right)\right\|^{2}+\frac{1}{2} \\
\frac{2 \widetilde{\omega} \widehat{\omega}}{\mu} & \geq \frac{\widetilde{\omega}^{2}}{\mu}-\frac{\omega^{2}}{\mu} \\
\widehat{E} \varepsilon & \leq|\widehat{E} \varepsilon| \leq \frac{\widehat{E}^{2}}{4}+\varepsilon_{M}{ }^{2}
\end{aligned}
$$

Also from Cauchy-Schwarz inequality

$$
\left\|\boldsymbol{W}_{\Phi}{ }^{* \mathrm{~T}} \boldsymbol{h}\left(\boldsymbol{X}_{\mathrm{in}}\right)\right\| \leq\left\|\boldsymbol{W}_{\Phi}{ }^{*}\right\|\left\|\boldsymbol{h}\left(\boldsymbol{X}_{\mathrm{in}}\right)\right\|
$$

Further

$$
\begin{aligned}
\widehat{E} \boldsymbol{W}_{\Phi}{ }^{* \mathrm{~T}} \boldsymbol{h}\left(\boldsymbol{X}_{\text {in }}\right) & \leq \frac{\widehat{E}^{2}}{2}\left\|\boldsymbol{W}_{\Phi}{ }^{*}\right\|^{2}\left\|\boldsymbol{h}\left(\boldsymbol{X}_{\text {in }}\right)\right\|^{2}+\frac{1}{2} \\
& =\frac{\widehat{E}^{2}}{2} \omega \boldsymbol{h}^{\mathrm{T}}\left(\boldsymbol{X}_{\text {in }}\right) \boldsymbol{h}\left(\boldsymbol{X}_{\text {in }}\right)+\frac{1}{2}
\end{aligned}
$$


Then (50) becomes

$$
\dot{L} \leq-\left(k_{2}-\frac{1}{4}\right) \widehat{E}^{2}-\frac{\widetilde{\omega}^{2}}{\mu}+\frac{1}{2}+\varepsilon_{M}^{2}+\frac{\omega^{2}}{\mu}
$$

Let $k_{2}>1 / 4$, and define the following compact sets:

$$
\begin{aligned}
& \Omega_{\widehat{E}}=\left\{\widehat{E}|| \widehat{E} \mid \leq \sqrt{\frac{\left(1 / 2+\varepsilon_{M}^{2}+\omega^{2} / \mu\right)}{\left(k_{2}-1 / 4\right)}}\right\} \\
& \Omega_{\widetilde{\omega}}=\left\{\widetilde{\omega}|| \widetilde{\omega} \mid \leq \sqrt{\frac{\left(1 / 2+\varepsilon_{M}^{2}+\omega^{2} / \mu\right)}{(1 / \mu)}}\right\}
\end{aligned}
$$

If $\widehat{E} \notin \Omega_{\widehat{E}}$ or $\widetilde{\omega} \notin \Omega_{\widetilde{\omega}}$, then $\dot{L}<0$. Thus these error signals $\widehat{E}$ and $\widetilde{\omega}$ are semiglobally uniformly ultimately bounded and can be invariant to the following sets $\Omega_{\widehat{E}}$ and $\Omega_{\widetilde{\omega}}$. The radiuses of $\Omega_{\widehat{E}}$ and $\Omega_{\widetilde{\omega}}$ can be made arbitrarily small by choosing appropriate design parameters, and the tracking errors $\widehat{E}$ and $\widetilde{\omega}$ can be arbitrarily small.

Substitute (46) into (19)

$$
E=\left[\begin{array}{ll}
\boldsymbol{\Lambda}^{\mathrm{T}} & 1
\end{array}\right] \boldsymbol{e}=\left[\begin{array}{ll}
\boldsymbol{\Lambda}^{\mathrm{T}} & 1
\end{array}\right](\widehat{\boldsymbol{e}}-\widetilde{\boldsymbol{e}})=\widehat{E}-\left[\begin{array}{ll}
\boldsymbol{\Lambda}^{\mathrm{T}} & 1
\end{array}\right] \widetilde{\boldsymbol{e}}
$$

Find the Euclid norm of (56) and combine the triangle inequality and the Cauchy-Schwarz inequality

$$
\begin{aligned}
\|E\| & =\left\|\widehat{E}-\left[\begin{array}{ll}
\boldsymbol{\Lambda}^{\mathrm{T}} & 1
\end{array}\right] \widetilde{\boldsymbol{e}}\right\| \leq\|\widehat{E}\|+\left\|\left[\begin{array}{ll}
\boldsymbol{\Lambda}^{\mathrm{T}} & 1
\end{array}\right] \widetilde{\boldsymbol{e}}\right\| \\
& \leq\|\widehat{E}\|+\left\|\left[\begin{array}{ll}
\boldsymbol{\Lambda}^{\mathrm{T}} & 1
\end{array}\right]\right\|\|\widetilde{\boldsymbol{e}}\|
\end{aligned}
$$

Let $c_{\lambda}=\left\|\left[\begin{array}{ll}\Lambda^{\mathrm{T}} & 1\end{array}\right]\right\|$, and substitute it into (47); then (57) can be written as

$$
\|E\| \leq\|\widehat{E}\|+c_{\lambda} \delta_{M}
$$

Therefore, $E$ and $e$ are also bounded, and the closed-loop control system is semiglobally uniformly ultimately bounded.

Theorem 12. Consider system (2) with external disturbances

$$
\begin{aligned}
& \dot{x}_{i}=f_{i}\left(\overline{\boldsymbol{x}}_{i}, x_{i+1}\right)+d_{i}(t), \quad 1 \leq i \leq n-1 \\
& \dot{x}_{n}=f_{n}\left(\overline{\boldsymbol{x}}_{n}, u\right)+d_{n}(t), \quad n \geq 2 \\
& y=x_{1}
\end{aligned}
$$

where $d_{i}(t)$ and $d_{n}(t)$ are unknown smooth bounded functions. Then for the nonaffine pure-feedback system (59), under the premise of Assumption 1, when the control law (42), the adaptive law (41), and the FD (45) are used, the closedloop control system is still semiglobally uniformly ultimately bounded.

Proof. The conversion of Steps $1 \sim n$ is performed on system (59), and the converted system is as follows:

$$
\begin{aligned}
& \dot{\zeta}_{i}=\zeta_{i+1}, \quad(i=1,2, \ldots, n-1) \\
& \dot{\zeta}_{n}=F_{n-1}\left(\bar{x}_{n}, u\right)+\sum L_{\forall D}\left(f_{i}\left(\overline{\boldsymbol{x}}_{i}, x_{i+1}\right)\right) \cdot d^{(\forall D)}(t)
\end{aligned}
$$

where $L_{\forall D}\left(f_{i}\left(\overline{\boldsymbol{x}}_{i}, x_{i+1}\right)\right)$ stands for a linear combination of any order derivatives of $f_{i}\left(\overline{\boldsymbol{x}}_{i}, x_{i+1}\right)$ and $d^{(\forall D)}(t)$ represents any order derivatives of $d_{i}(t)$ or $d_{n}(t)$.

Because $f_{i}\left(\overline{\boldsymbol{x}}_{i}, x_{i+1}\right), d_{i}(t)$ and $d_{n}(t)$ are all smooth and bounded functions, and they are infinitely derivable in the domain of definition, $L_{\forall D}\left(f_{i}\left(\overline{\boldsymbol{x}}_{i}, x_{i+1}\right)\right)$ and $d^{(\forall D)}(t)$ are bounded, and there is

$$
\left|\sum L_{\forall D}\left(f_{i}\left(\overline{\boldsymbol{x}}_{i}, x_{i+1}\right)\right) \cdot d^{(\forall D)}(t)\right| \leq \wp_{M}
$$

where $\wp_{M} \in \mathbf{R}^{+}$is its upper bound.

Alike the proof process of Theorem 11, in the above case, (54) becomes

$$
\dot{L}^{*} \leq-\left(k_{2}-\frac{1}{4}\right) \widehat{E}^{2}-\frac{\widetilde{\omega}^{2}}{\mu}+\frac{1}{2}+\varepsilon_{M}{ }^{2}+\frac{\omega^{2}}{\mu}+\wp_{M}
$$

Change the original compact sets to

$$
\begin{aligned}
& \Omega_{\widehat{E}}{ }^{*}=\left\{\widehat{E}|| \widehat{E} \mid \leq \sqrt{\frac{\left(1 / 2+\varepsilon_{M}^{2}+\omega^{2} / \mu+\wp_{M}\right)}{\left(k_{2}-1 / 4\right)}}\right\} \\
& \Omega_{\widetilde{\omega}}{ }^{*}=\left\{\widetilde{\omega}|| \widetilde{\omega} \mid \leq \sqrt{\frac{\left(1 / 2+\varepsilon_{M}^{2}+\omega^{2} / \mu+\wp_{M}\right)}{(1 / \mu)}}\right\}
\end{aligned}
$$

It can also be proved that error signals $\widehat{E}$ and $\widetilde{\omega}$ are semiglobally uniformly ultimately bounded and can be invariant to the following set $\Omega_{\widehat{E}}$ and $\Omega_{\widetilde{\omega}}$. Furthermore, $E$ and $e$ are bounded, and the closed-loop control system is still semiglobally uniformly ultimately bounded.

Theorem 12 shows that when the system is affected by external disturbances, the controller designed in this paper can still theoretically guarantee the stability of the closed-loop control system.

At this point, the entire process of the control law design is completed. The block diagram of the designed control system is shown in Figure 2.

\section{Simulation Example}

In order to verify the effectiveness of the proposed controller, we apply it to the control of the longitudinal movement of the air-breathing hypersonic vehicle (AHV). Consider the following AHV parameter fitting model [28] established by Parker, a US Air Force researcher.

$$
\begin{aligned}
& \dot{V}=\frac{T \cos (\theta-\gamma)-D}{m}-g \sin \gamma \\
& \dot{h}=V \sin \gamma \\
& \dot{\gamma}=\frac{L+T \sin (\theta-\gamma)}{m V}-\frac{g}{V} \cos \gamma \\
& \dot{\theta}=Q
\end{aligned}
$$




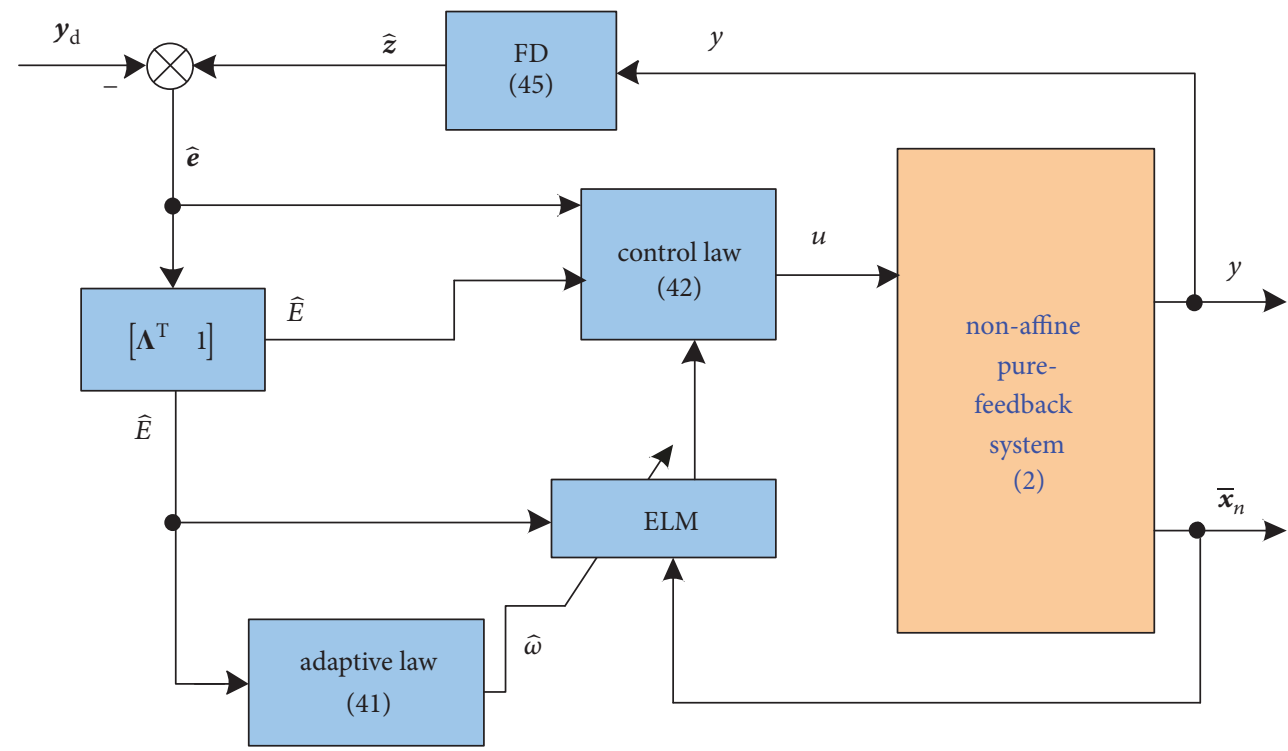

FIGURE 2: The block diagram of the designed control system.

$$
\begin{aligned}
\dot{Q} & =\frac{M+\widetilde{\psi}_{1} \ddot{\eta}_{1}+\widetilde{\psi}_{2} \ddot{\eta}_{2}}{I_{\mathrm{yy}}} \\
k_{1} \ddot{\eta}_{1} & =-2 \zeta_{1} \omega_{1} \dot{\eta}_{1}-\omega_{1}^{2} \eta_{1}+N_{1}-\widetilde{\psi}_{1} \frac{M}{I_{\mathrm{yy}}}-\frac{\widetilde{\psi}_{1} \widetilde{\psi}_{2} \ddot{\eta}_{2}}{I_{\mathrm{yy}}} \\
k_{2} \ddot{\eta}_{2} & =-2 \zeta_{2} \omega_{2} \dot{\eta}_{2}-\omega_{2}^{2} \eta_{2}+N_{2}-\widetilde{\psi}_{2} \frac{M}{I_{\mathrm{yy}}}-\frac{\widetilde{\psi}_{2} \widetilde{\psi}_{1} \ddot{\eta}_{1}}{I_{\mathrm{yy}}}
\end{aligned}
$$

where velocity $V$, altitude $h$, flight-path $\gamma$, pitch angle $\theta$, and pitch rate $Q$ are the rigid-body states; $m$ and $I_{\mathrm{yy}}$ stand for vehicle mass and moment of inertia, respectively; the flexible states are $\eta_{1}$ and $\eta_{2}, \zeta_{i}$ and $\omega_{i}(i=1,2)$ represent damping ratio and natural frequency for flexible modes, respectively; $N_{1}$ and $N_{2}$ are the generalized forces. The approximations of thrust $T$, drag $D$, lift $L$, and pitching moment $M$ are expressed as [28]

$$
\begin{aligned}
& T \approx C_{T}^{\alpha^{3}} \alpha^{3}+C_{T}^{\alpha^{2}} \alpha^{2}+C_{T}^{\alpha} \alpha+C_{T}^{0} \\
& D \approx \bar{q} S\left(C_{D}^{\alpha^{2}} \alpha^{2}+C_{D}^{\alpha} \alpha+C_{D}^{\delta_{\mathrm{e}}^{2}} \delta_{\mathrm{e}}^{2}+C_{D}^{\delta_{\mathrm{e}}} \delta_{\mathrm{e}}+C_{D}^{0}\right) \\
& L \approx \bar{q} S\left(C_{L}^{\alpha} \alpha+C_{L}^{\delta_{\mathrm{e}}} \delta_{\mathrm{e}}+C_{L}^{0}\right) \\
& M \approx z_{T} T+\bar{q} S \bar{c}\left[C_{M, \alpha}^{\alpha^{2}} \alpha^{2}+C_{M, \alpha}^{\alpha} \alpha+C_{M, \alpha}^{0}+c_{\mathrm{e}} \delta_{\mathrm{e}}\right]
\end{aligned}
$$

with

$$
\begin{aligned}
& C_{T}^{\alpha^{3}}=\beta_{1}(h, \bar{q}) \Phi+\beta_{2}(h, \bar{q}) \\
& C_{T}^{\alpha^{2}}=\beta_{3}(h, \bar{q}) \Phi+\beta_{4}(h, \bar{q}) \\
& C_{T}^{\alpha}=\beta_{5}(h, \bar{q}) \Phi+\beta_{6}(h, \bar{q}) \\
& C_{T}^{0}=\beta_{7}(h, \bar{q}) \Phi+\beta_{8}(h, \bar{q})
\end{aligned}
$$

$$
\begin{aligned}
& \bar{q}=\frac{1}{2} \bar{\rho} V^{2} \\
& \bar{\rho}=\bar{\rho}_{0} \exp \left(\frac{h_{0}-h}{h_{\mathrm{s}}}\right)
\end{aligned}
$$

where $\alpha=\theta-\gamma$ is the angle of attack; fuel equivalence ratio $\Phi$ and elevator angular deflection $\delta_{\mathrm{e}}$ are control inputs. The meanings, expressions, and specific values of other related physical quantities of the above AHV model can be found in [28].

In order to facilitate the control law design, according to [29], the AHV model is first decomposed formally into a velocity subsystem (64) controlled mainly by $\Phi$ and an altitude subsystem (65)-(68) controlled mainly by $\delta_{\mathrm{e}}$. Then use the control strategy of this paper to design the control laws $\Phi$ and $\delta_{\mathrm{e}}$ as

$$
\begin{aligned}
\widetilde{V} & =V-V_{\text {ref }} \\
\Phi & =k_{V}^{-1}\left(\Phi_{0}-\frac{1}{2} \widetilde{V} \widehat{\varphi}_{1} \boldsymbol{h}_{1}^{\mathrm{T}}\left(\boldsymbol{X}_{1}\right) \boldsymbol{h}_{1}\left(\boldsymbol{X}_{1}\right)\right) \\
\Phi_{0} & =-k_{V 1} \widetilde{V}-k_{V 2} \int_{0}^{t} \widetilde{V} \mathrm{~d} \tau+\dot{V}_{\text {ref }} \\
\dot{\hat{\varphi}}_{1} & =\frac{\lambda_{1}}{2} \widetilde{V}^{2} \boldsymbol{h}_{1}^{\mathrm{T}}\left(\boldsymbol{X}_{1}\right) \boldsymbol{h}_{1}\left(\boldsymbol{X}_{1}\right)-2 \widehat{\varphi}_{1} \\
\tilde{h} & =h-h_{\text {ref }} \\
\gamma_{\mathrm{d}} & =\arcsin \left(\frac{-k_{\gamma} \tilde{h}+\dot{h}_{\text {ref }}}{V}\right) \\
e_{0} & =\gamma-\gamma_{\mathrm{d}} \\
\dot{\hat{e}}_{0} & =\dot{\hat{\gamma}}-\dot{\hat{\gamma}}_{\mathrm{d}}
\end{aligned}
$$




$$
\begin{aligned}
& \ddot{\hat{e}}_{0}=\ddot{\hat{\gamma}}-\ddot{\hat{\gamma}}_{\mathrm{d}} \\
& \widehat{E}=\ddot{\widehat{e}}_{0}+3 \mu \dot{\hat{e}}_{0}+3 \mu^{2} e_{0}+\mu^{3} \int_{0}^{t} e_{0} \mathrm{~d} \tau \\
& \delta_{\mathrm{e}}=k_{h}^{-1}\left(\delta_{\mathrm{e} 0}-\frac{1}{2} \widehat{E} \widehat{\varphi}_{2} \boldsymbol{h}_{2}^{\mathrm{T}}\left(\boldsymbol{X}_{2}\right) \boldsymbol{h}_{2}\left(\boldsymbol{X}_{2}\right)\right) \\
& \delta_{\mathrm{e} 0}=-k_{h 1} \widehat{E}+\ddot{\hat{\gamma}}_{\mathrm{d}}-3 \mu \ddot{\vec{e}}_{0}-3 \mu^{2} \dot{\hat{e}}_{0}-\mu^{3} e_{0} \\
& \dot{\hat{\varphi}}_{2}=\frac{\lambda_{2}}{2} \widehat{E}^{2} \boldsymbol{h}_{2}^{\mathrm{T}}\left(\boldsymbol{X}_{2}\right) \boldsymbol{h}_{2}\left(\boldsymbol{X}_{2}\right)-2 \widehat{\varphi}_{2}
\end{aligned}
$$

where $V_{\text {ref }}$ and $h_{\text {ref }}$ are the reference commands and $\boldsymbol{X}_{1}=$ $\left[V, \Phi_{0}\right]^{\mathrm{T}} \in \mathbf{R}^{2}$ and $\boldsymbol{X}_{2}=\left[\gamma, \theta, Q, \delta_{\mathrm{e} 0}\right]^{\mathrm{T}} \in \mathbf{R}^{4}$ stand for the ELM input vectors. Using FD to estimate the differential signals in the above equations

$$
\begin{aligned}
& (\widehat{\gamma})^{\prime}=\dot{\hat{\gamma}} \\
& (\dot{\hat{\gamma}})^{\prime}=\ddot{\widehat{\gamma}} \\
& (\ddot{\hat{\gamma}})^{\prime}=\ddot{\widehat{\gamma}} \\
& (\ddot{\hat{\gamma}})^{\prime}=R_{1}^{4}\left[-a_{11} \arctan (\widehat{\gamma}-\gamma)-a_{12} \arctan \left(\frac{\dot{\hat{\gamma}}}{R_{1}}\right)\right. \\
& \left.-a_{13} \arctan \left(\frac{\ddot{\hat{\gamma}}}{R_{1}^{2}}\right)-a_{14} \arctan \left(\frac{\ddot{\hat{\gamma}}}{R_{1}^{3}}\right)\right] \\
& \left(\widehat{\gamma}_{\mathrm{d}}\right)^{\prime}=\dot{\widehat{\gamma}}_{\mathrm{d}} \\
& \left(\dot{\widehat{\gamma}}_{\mathrm{d}}\right)^{\prime}=\ddot{\widehat{\gamma}}_{\mathrm{d}} \\
& \left(\ddot{\widehat{\gamma}}_{\mathrm{d}}\right)^{\prime}=\ddot{\widehat{\hat{\gamma}}}_{\mathrm{d}} \\
& \left(\ddot{\widehat{\gamma}}_{\mathrm{d}}\right)^{\prime}=R_{2}^{4}\left[-a_{21} \arctan \left(\widehat{\gamma}_{\mathrm{d}}-\gamma_{\mathrm{d}}\right)-a_{22} \arctan \left(\frac{\dot{\hat{\gamma}}_{\mathrm{d}}}{R_{2}}\right)\right. \\
& \left.-a_{23} \arctan \left(\frac{\ddot{\hat{\gamma}}_{\mathrm{d}}}{R_{1}^{2}}\right)-a_{24} \arctan \left(\frac{\ddot{\widehat{\gamma}}_{\mathrm{d}}}{R_{2}^{3}}\right)\right]
\end{aligned}
$$

where $R_{i}$ and $a_{i j}(i=1,2 ; j=1,2,3,4) \in \mathbf{R}^{+}$are design parameters; $\widehat{\gamma}^{(n)}$ and $\widehat{\gamma}_{\mathrm{d}}^{(n)}(n=0,1,2,3)$ represent the estimation of $\gamma^{(n)}$ and $\gamma_{\mathrm{d}}{ }^{(n)}$, respectively.

With AHV longitudinal motion model as the controlled object, the tracking simulation of the velocity and altitude reference commands is performed. The initial velocity and initial altitude of AHV are chosen as $V=2500 \mathrm{~m} / \mathrm{s}$ and $h=27000 \mathrm{~m}$. The velocity step and altitude step are chosen as $\Delta V=200 \mathrm{~m} / \mathrm{s}$ and $\Delta h=200 \mathrm{~m}$. Both the velocity and altitude reference inputs are given by a second-order reference model with a damping ratio of 0.9 and a natural frequency of $0.1 \mathrm{rad} / \mathrm{s}$ [24]. The design parameters of controller are chosen as $k_{V}=5, k_{V 1}=0.3, k_{V 2}=0.8, k_{\gamma}=2, k_{h}=0.9, k_{h 1}=50$, and $\mu=7$. The design parameters of the adaptive law are taken as $\lambda_{1}=\lambda_{2}=0.05$. The design parameters of the FD are chosen as $R_{1}=R_{2}=0.05, a_{11}=a_{13}=a_{21}=a_{23}=0.5$,

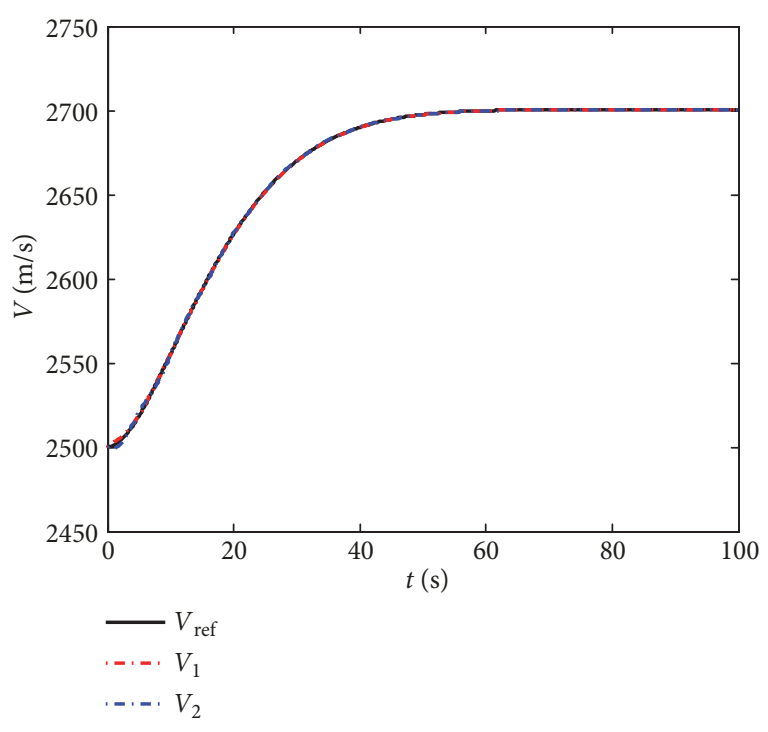

FIgURE 3: Velocity tracking.

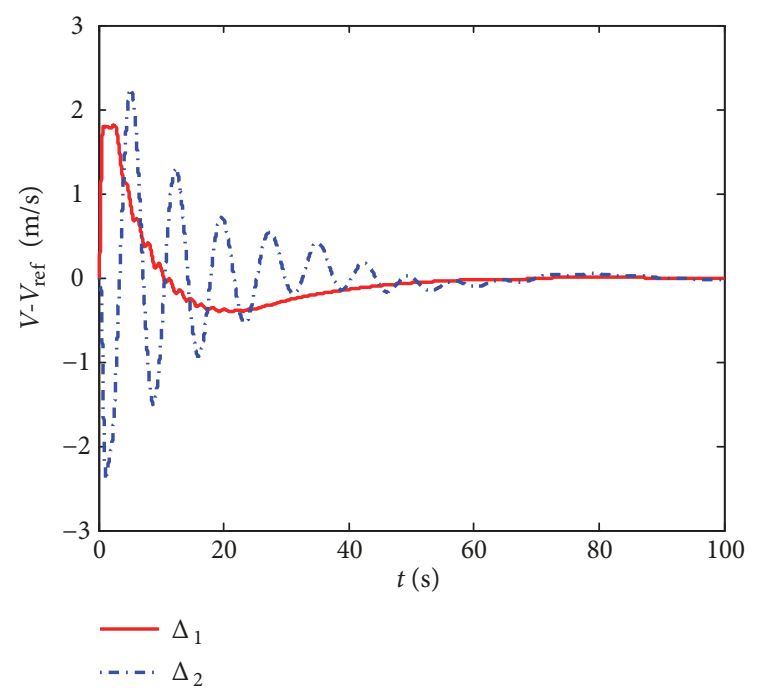

FIGURE 4: Velocity tracking error.

$a_{12}=a_{14}=a_{22}=a_{24}=0.1$. The node number of ELM is chosen as 20. The hidden layer node is set as additive Sigmoid activation function; hidden layer node parameters $\left(\boldsymbol{\omega}_{i}, b_{i}\right)$ are randomly given in $[-1,1]$ and $[0,1]$, respectively. To verify the robustness of the controller, it is assumed that there is a perturbation of $\pm 40 \%$ for the aerodynamic coefficient of the AHV model, which is expressed as $C=$ $C_{0}[1+0.4 \sin (0.1 \pi t)]$, where $C_{0}$ represents nominal value and $C$ stands for simulation value. And after $50 \mathrm{~s}$, add external disturbances $d_{1}=2 \sin (0.1 \pi t)$ and $d_{2}=0.02 \sin (0.1 \pi t)$ to (64) and (68) in AHV model, respectively [24]. The subscript " 1 " in the Figures 3 to 11 shows the result obtained by the proposed method and the subscript " 2 " shows the result obtained by the method of [19].

The simulation results show that for the AHV longitudinal motion model, when there are parametric perturbations and external disturbances, both the adaptive neural control 

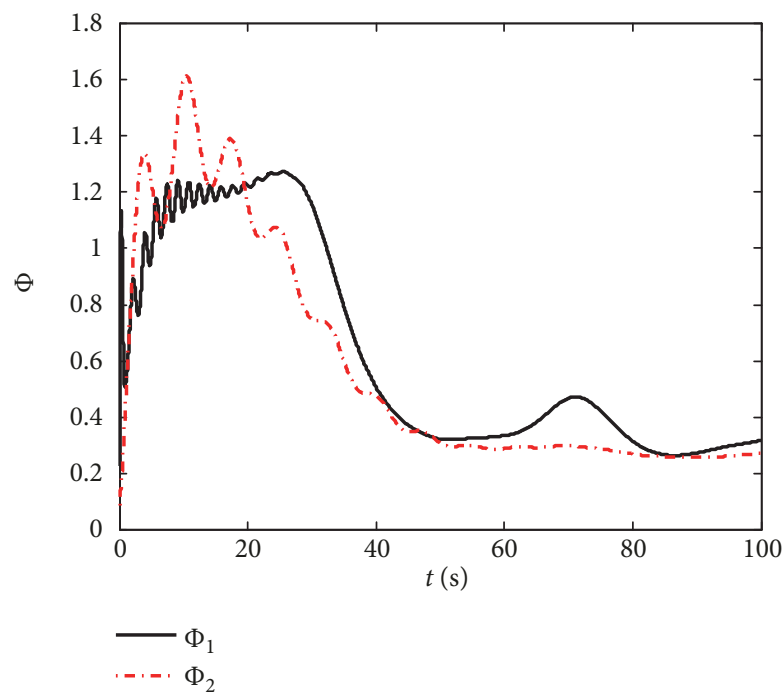

FIGURE 5: Fuel equivalence ratio.

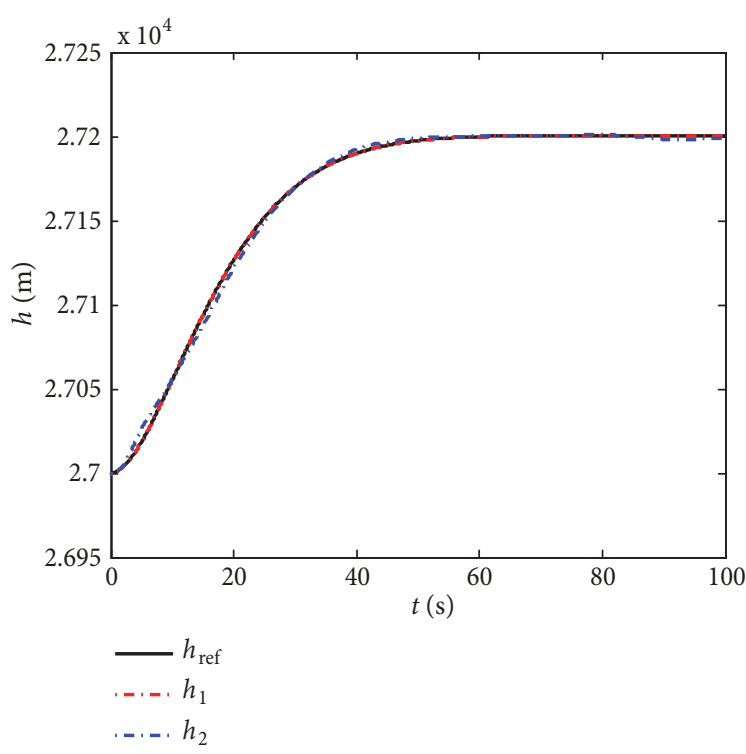

FIgURE 6: Altitude tracking.

law designed in this paper and the control method proposed in [19] can guarantee that the velocity $V$ and altitude $h$ track their reference commands effectively (see Figures 3 and 6). However, for a strongly nonlinear, strongly coupled, and fast time-varying system such as AHV, the control accuracy of the control method proposed in this paper is significantly higher than that in [19] (see Figures 4 and 7). At the same time, under the control method of this paper, the variation range of AHV's state variables $\gamma, \theta, Q$ and control inputs $\Phi$ and $\delta_{\mathrm{e}}$ are smaller than that of the control method in [19], which better meets the requirements of AHV's flight envelope [30] (see Figures 5,8 , and 9-11).

\section{Conclusion}

(1) In this paper, a novel adaptive neural controller is designed for nonaffine pure-feedback system. Under the

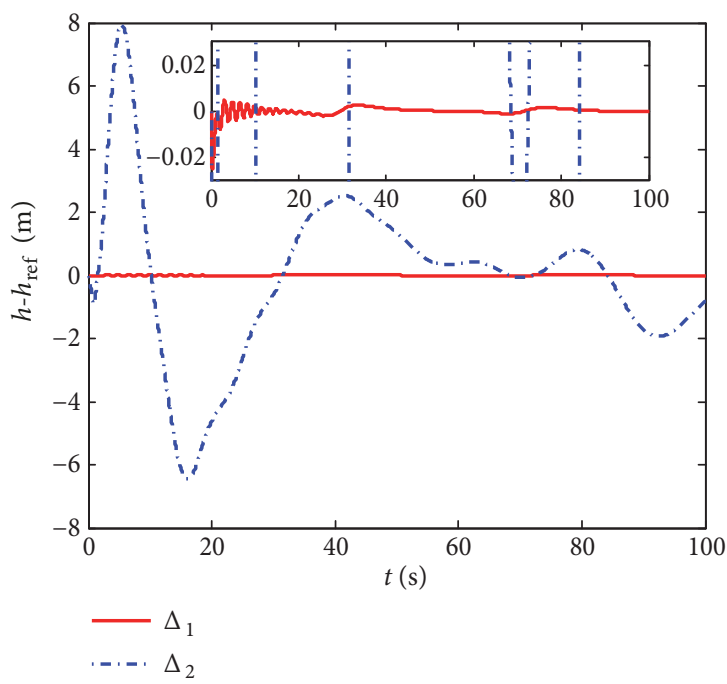

FIGURE 7: Altitude tracking error.

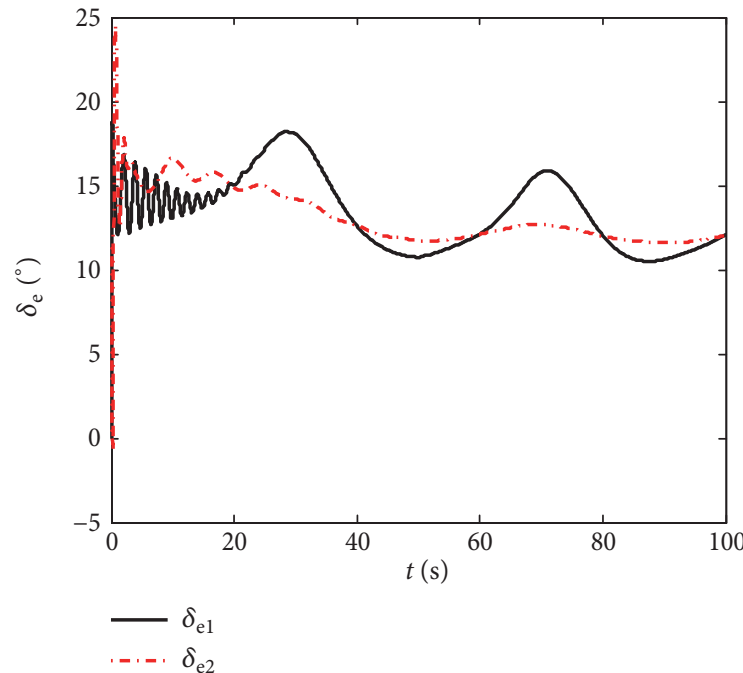

FIgURE 8: Elevator angular deflection.

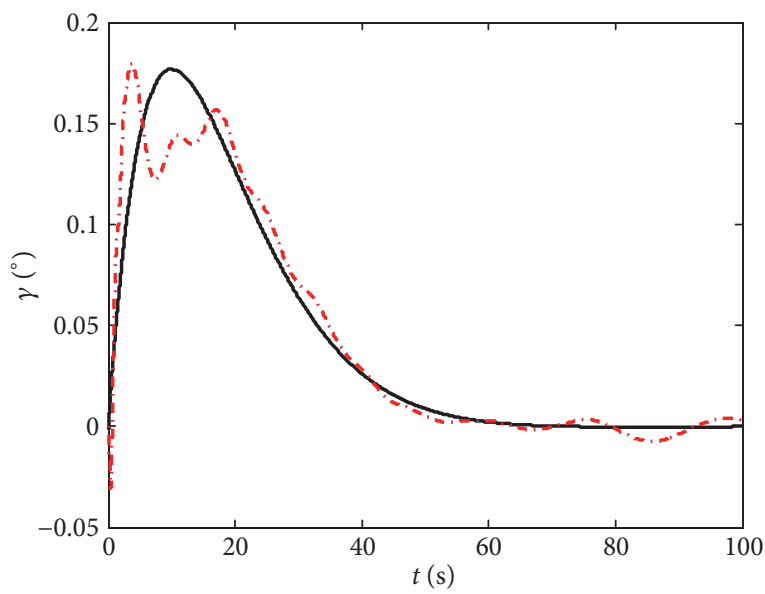

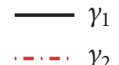

FIGURE 9: Flight-path. 


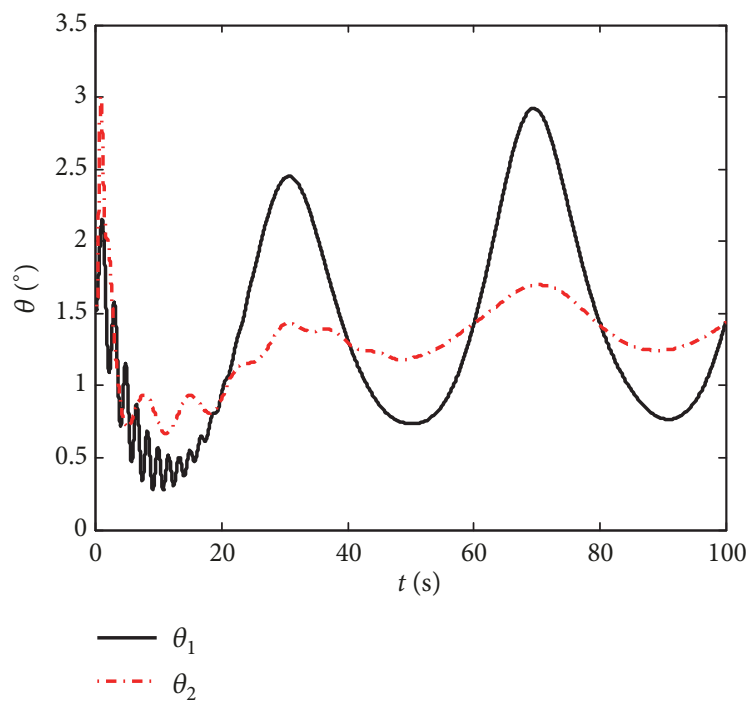

Figure 10: Pitch angle.

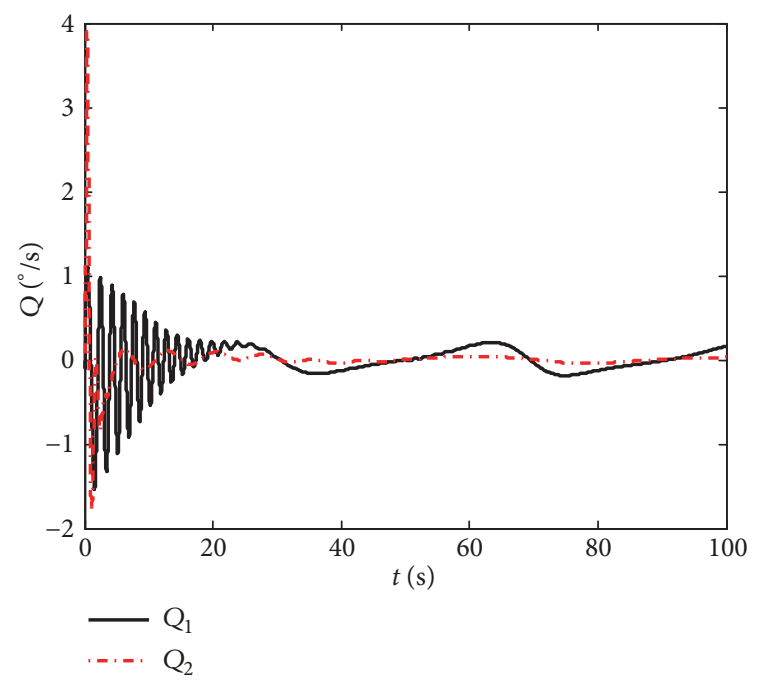

Figure 11: Pitch rate.

premise of ensuring the stability and tracking accuracy of the closed-loop control system, the derivation process is simpler and the control law form is more concise than the controller designed indirectly based on the backstepping method.

(2) The method in this paper is a nonaffine control method for a more realistic nonaffine model, which has higher reliability and practical value than the traditional affine control method.

(3) The adopted ELM algorithm simplifies the process of adjusting the network training parameters.

(4) When the control method of this paper is applied to AHV, the simulation results show that the control method designed in this paper has a higher control precision and stronger robustness than the traditional control method.

\section{Data Availability}

The data sources from the references are all marked in this paper. And the parameters designed are all described in this paper. Therefore, the data in this paper has been fully covered and can be obtained.

\section{Conflicts of Interest}

The authors declare that they have no conflicts of interest.

\section{Acknowledgments}

This work was supported by the National Natural Science Foundation of China (Grant no. 61703421) and the Aeronautical Science Foundation of China (Grant no. 20150096006).

\section{References}

[1] Y. G. Hong, "Remarks on the development of nonlinear systems and control theory," Journal of Systems Science and Mathematical Sciences, vol. 34, no. 11, pp. 1283-1290, 2014.

[2] M. Krstic, I. Kanellakopoulous, and V. Kokotovic P, Nonlinear and Adaptive Control Design, John Wiley \& Sons, New York, NY, USA, 1995.

[3] S. Ge, T. Lee, and J. Wang, "Adaptive control of non-affine nonlinear systems using neural networks," in Proceedings of the 15th IEEE International Symposium on Intelligent Control, pp. 13-18, IEEE, Rio Patras, Greece, 2000.

[4] C.-H. Cheng, Y.-A. Hu, and J.-H. Wu, "Auto disturbance controller of non-affine nonlinear pure feedback systems," Acta Automatica Sinica, vol. 40, no. 7, pp. 1528-1536, 2014 (Chinese).

[5] R. M. Sanner and J. E. Slotine, "Gaussian networks for direct adaptive control," IEEE Transactions on Neural Networks and Learning Systems, vol. 3, no. 6, pp. 837-863, 1992.

[6] M. M. Polycarpou, "Stable adaptive neural control scheme for nonlinear systems," IEEE Transactions on Automatic Control, vol. 41, no. 3, pp. 447-451, 1996.

[7] S. S. Ge, C. C. Hang, and T. Zhang, "Nonlinear adaptive control using neural networks and its application to CSTR systems," Journal of Process Control, vol. 9, no. 4, pp. 313-323, 1999.

[8] T. Zhang, S. S. Ge, and C. C. Hang, "Adaptive neural network control for strict-feedback nonlinear systems using backstepping design," Automatica, vol. 36, no. 12, pp. 1835-1846, 2000.

[9] S. S. Ge and C. Wang, "Adaptive neural control of uncertain MIMO nonlinear systems," IEEE Transactions on Neural Networks and Learning Systems, vol. 15, no. 3, pp. 674-692, 2004.

[10] Y. Li, S. Qiang, X. Zhuang, and O. Kaynak, "Robust and adaptive backstepping control for nonlinear systems using RBF neural networks," IEEE Transactions on Neural Networks and Learning Systems, vol. 15, no. 3, pp. 693-701, 2004.

[11] W. Chen, S. S. Ge, J. Wu, and M. Gong, "Globally stable adaptive backstepping neural network control for uncertain strict-feedback systems with tracking accuracy known a priori," IEEE Transactions on Neural Networks and Learning Systems, vol. 26, no. 9, pp. 1842-1854, 2015.

[12] S. S. Ge, C. Yang, and T. H. Lee, "Adaptive predictive control using neural network for a class of pure-feedback systems in discrete time," IEEE Transactions on Neural Networks and Learning Systems, vol. 19, no. 9, pp. 1599-1614, 2008.

[13] J.-H. Park, S.-H. Kim, and C.-J. Moon, "Adaptive neural control for strict-feedback nonlinear systems without backstepping," IEEE Transactions on Neural Networks and Learning Systems, vol. 20, no. 7, pp. 1204-1209, 2009. 
[14] L. Jun and S. Qing, "Adaptive control for a class of uncertain pure-feedback nonlinear systems using Backstepping based on extreme learning machine," CIESC Journal, vol. 67, no. 7, pp. 2934-2942, 2016 (Chinese).

[15] S. S. Ge and C. Wang, "Adaptive NN control of uncertain nonlinear pure-feedback systems," Automatica, vol. 38, no. 4, pp. 671-682, 2002.

[16] D. Wang and J. Huang, "Adaptive neural network control for a class of uncertain nonlinear systems in pure-feedback form," Automatica, vol. 38, no. 8, pp. 1365-1372, 2002.

[17] B. H. Du and S. J. Li, "Adaptive neural network variable structure control for a class of non-affine nonlinear pure-feedback systems," Journal of System Engineering and Electronics, vol. 30, no. 4, pp. 723-726, 2008 (Chinese).

[18] J. Zhang, Q. Zhu, X. Wu, and Y. Li, "A generalized indirect adaptive neural networks backstepping control procedure for a class of non-affine nonlinear systems with pure-feedback prototype," Neurocomputing, vol. 121, no. 9, pp. 131-139, 2013.

[19] Y. Hu A, C. Cheng H, and Q. Zou, "Indirect adaptive neural networks controller for non-affine pure-feedback systems," Control Theory \& Application, vol. 31, no. 4, pp. 467-478, 2014 (Chinese).

[20] G. Huang, L. Chen, and C. Siew, "Universal approximation using incremental constructive feedforward networks with random hidden nodes," IEEE Transactions on Neural Networks and Learning Systems, vol. 17, no. 4, pp. 879-892, 2006.

[21] G. Huang, D. H. Wang, and Y. Lan, "Extreme learning machines: a survey," International Journal of Machine Learning and Cybernetics, vol. 2, no. 2, pp. 107-122, 2011.

[22] J. Li and Y.-Q. Nai, "Robust adaptive control for a class of MIMO affine nonlinear systems using extreme learning machine," Control and Decision, vol. 30, no. 9, pp. 1559-1566, 2015 (Chinese).

[23] Y. Elkoteshy, L. C. Jiao, and W. Chen, "ELM-based adaptive Backstepping neural control for a class of uncertain MIMO nonlinear systems with predefined tracking accuracy," International Journal of Control, vol. 87, no. 5, pp. 1047-1060, 2014.

[24] C. Luo, H. Lei, D. Zhang, and X. Zou, "Adaptive neural control of hypersonic vehicles with actuator constraints," International Journal of Aerospace Engineering, vol. 2018, Article ID 1284753, 15 pages, 2018.

[25] J.-H. Park, S.-H. Huh, S.-H. Kim, S.-J. Seo, and G.-T. Park, "Direct adaptive controller for nonaffine nonlinear systems using self-structuring neural networks," IEEE Transactions on Neural Networks and Learning Systems, vol. 16, no. 2, pp. 414422, 2005.

[26] A. J. Calise, N. Hovakimyan, and M. Idan, "Adaptive output feedback control of nonlinear systems using neural networks," Automatica, vol. 37, no. 1, pp. 1201-1211, 2001.

[27] X. Wang, Z. Chen, and G. Yang, "Finite-time-convergent differentiator based on singular perturbation technique," IEEE Transactions on Automatic Control, vol. 52, no. 9, pp. 1731-1737, 2007.

[28] J. T. Parker, A. Serrani, S. Yurkovich, M. A. Bolender, and D. B. Doman, "Control-oriented modeling of an air-breathing hypersonic vehicle," Journal of Guidance, Control, and Dynamics, vol. 30, no. 3, pp. 856-869, 2007.

[29] L. Fiorentini and A. Serrani, "Adaptive restricted trajectory tracking for a non-minimum phase hypersonic vehicle model," Automatica, vol. 48, no. 7, pp. 1248-1261, 2012.
[30] L. Fiorentini, Nonlinear Adaptive Controller Design for AirBreathing Hypersonic Vehicles, The Ohio State University, Columbus, OH, USA, 2010. 


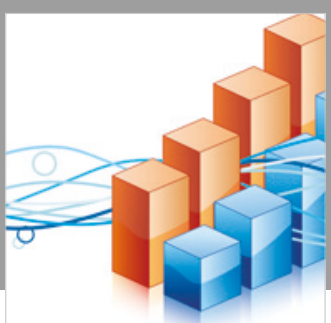

Advances in

Operations Research

\section{-n-m}
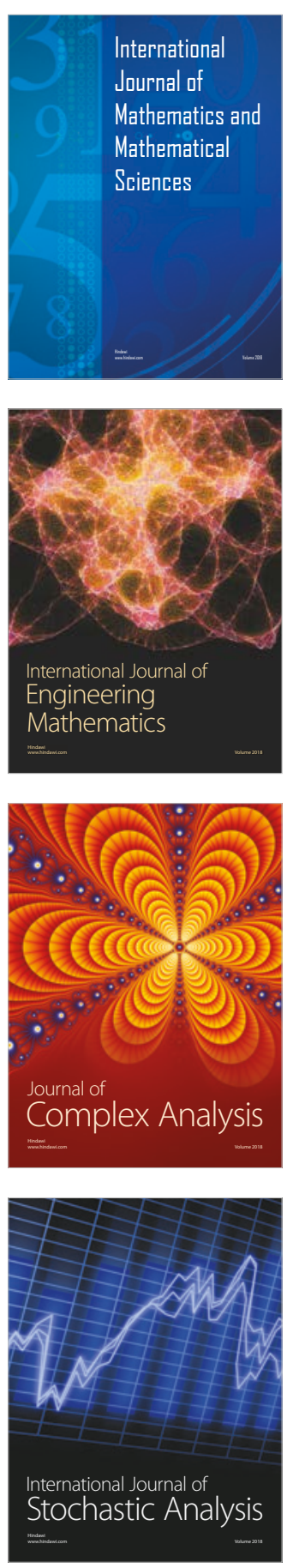
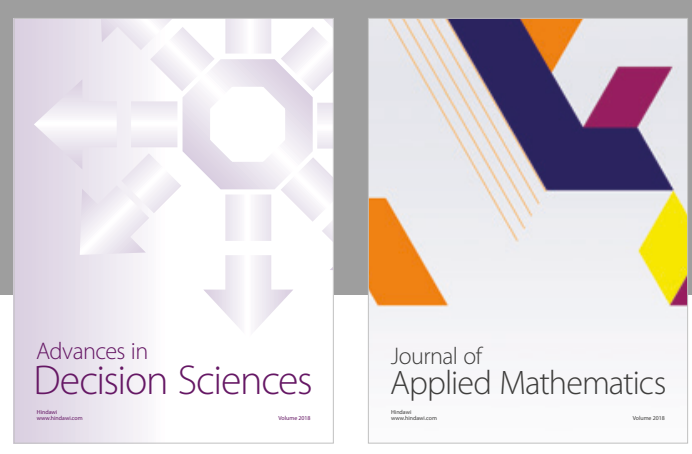

Journal of

Applied Mathematics
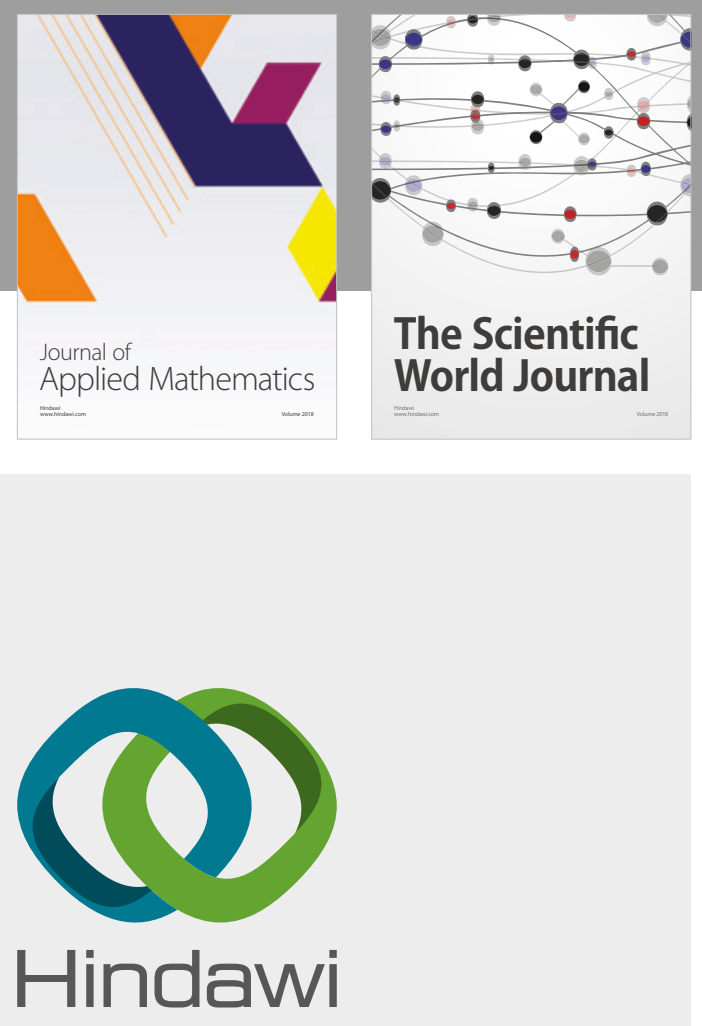

Submit your manuscripts at

www.hindawi.com

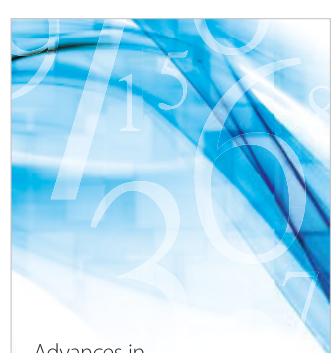

Advances in
Numerical Analysis
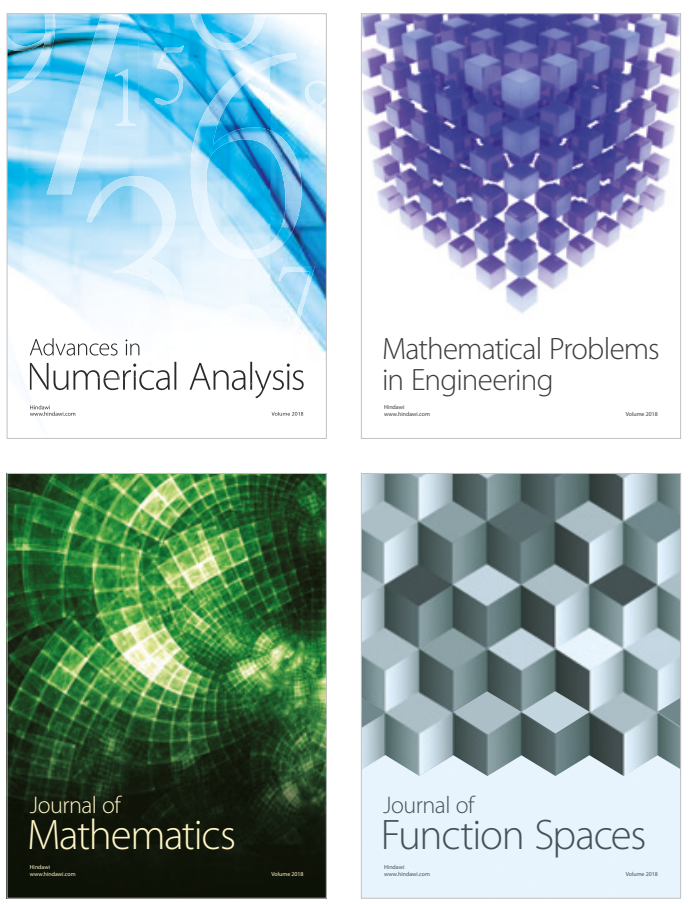

Mathematical Problems in Engineering

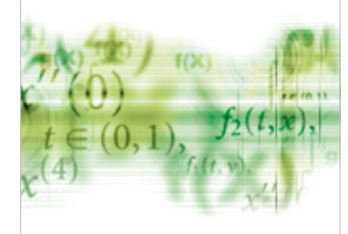

International Journal of

Differential Equations

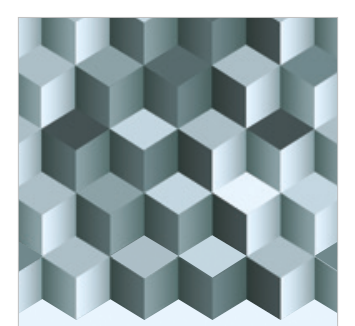

Journal of

Function Spaces

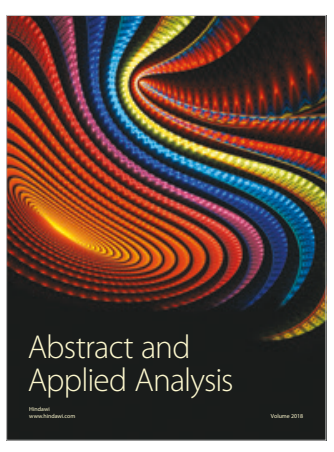

The Scientific

World Journal

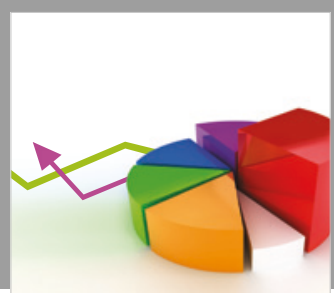

Journal of

Probability and Statistics
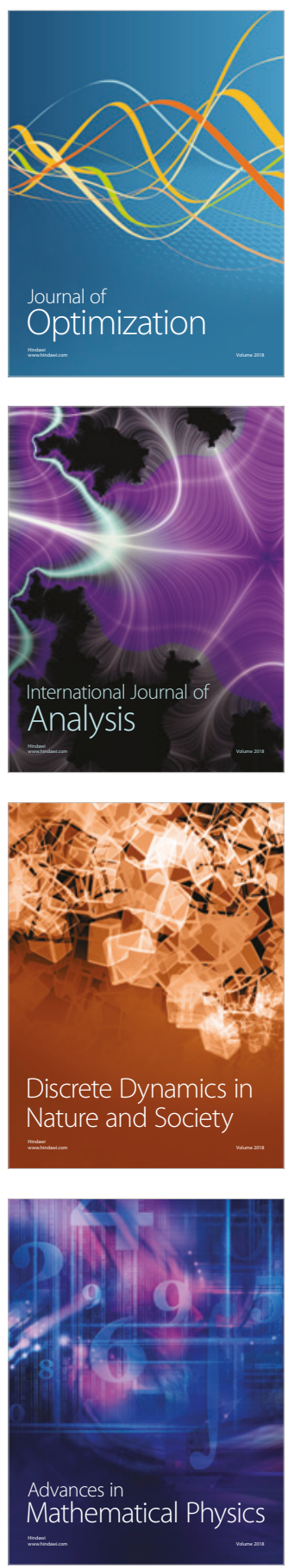